\title{
Mass ejection from disks surrounding a low-mass black hole: Viscous neutrino-radiation hydrodynamics simulation in full general relativity
}

\author{
Sho Fujibayashi@, ${ }^{1, *}$ Masaru Shibata ${ }^{1,2}$ Shinya Wanajo ${ }^{1,3}$ Kenta Kiuchi $\odot,{ }^{1,2}$ \\ Koutarou Kyutoku $\odot,{ }^{4,5,3,2}$ and Yuichiro Sekiguchi $\odot^{6}$ \\ ${ }^{1}$ Max Planck Institute for Gravitational Physics (Albert Einstein Institute), \\ Am Muhlenberg 1, Potsdam-Golm 14476, Germany \\ ${ }^{2}$ Center for Gravitational Physics, Yukawa Institute for Theoretical Physics, \\ Kyoto University, Kyoto 606-8502, Japan \\ ${ }^{3}$ Interdisciplinary Theoretical and Mathematical Science (iTHEMS) Research Group, \\ RIKEN, Wako, Saitama 351-0198, Japan \\ ${ }^{4}$ Department of Physics, Kyoto University, Kyoto 606-8502, Japan \\ ${ }^{5}$ Department of Particle and Nuclear Physics, the Graduate University for Advanced Studies (Sokendai), \\ Tsukuba 305-0801, Japan \\ ${ }^{6}$ Department of Physics, Toho University, Funabashi, Chiba 274-8510, Japan
}

(Received 15 January 2020; accepted 2 March 2020; published 24 April 2020)

\begin{abstract}
New viscous neutrino-radiation hydrodynamics simulations are performed for accretion disks surrounding a spinning black hole with low mass $3 M_{\odot}$ and dimensionless spin 0.8 or 0.6 in full general relativity, aiming at modeling the evolution of a merger remnant of massive binary neutron stars or low-mass black hole-neutron star binaries. We reconfirm the following results found by previous studies of other groups: $15 \%-30 \%$ of the disk mass is ejected from the system with the average velocity of $\sim 5 \%-10 \%$ of the speed of light for the plausible profile of the disk as merger remnants. In addition, we find that for the not extremely high viscous coefficient case, the neutron richness of the ejecta does not become very high, because weak interaction processes enhance the electron fraction during the viscous expansion of the disk before the onset of the mass ejection, resulting in the suppression of the lanthanide synthesis. For high-mass disks, the viscous expansion timescale is increased by a longer-term neutrino emission, and hence, the electron fraction of the ejecta becomes even higher. We also confirm that the mass distribution of the electron fraction depends strongly on the magnitude of the given viscous coefficient. This demonstrates that a first-principle magnetohydrodynamics simulation is necessary for black hole-disk systems with sufficient grid resolution and with sufficiently long timescale (longer than seconds) to clarify the nucleosynthesis and electromagnetic signals from them.
\end{abstract}

DOI: 10.1103/PhysRevD.101.083029

\section{INTRODUCTION}

The first direct detection of gravitational waves from the final stage of an inspiraling binary neutron star system (GW170817) by advanced LIGO and advanced VIRGO [1] was accompanied with a wide variety of the follow-up observations of electromagnetic counterparts [2]. This event heralded the opening of the era of multimessenger astronomy composed of gravitational-wave and electromagnetic-counterpart observations, and it demonstrated

\footnotetext{
*sho.fujibayashi@aei.mpg.de
}

Published by the American Physical Society under the terms of the Creative Commons Attribution 4.0 International license. Further distribution of this work must maintain attribution to the author(s) and the published article's title, journal citation, and DOI. Open access publication funded by the Max Planck Society. that the observation of electromagnetic signals plays a key role for understanding the merger and subsequent mass ejection processes of neutron-star binaries, which cannot be understood only from gravitational-wave observation.

A popular interpretation for the merger and postmerger evolution of binary neutron stars in GW170817 is as follows (e.g., see Refs. [3-6]): After the merger of a binary neutron star, a hypermassive neutron star was formed with an accretion disk around it. Subsequently, the hypermassive neutron star survived for $\sim 0.1-1 \mathrm{~s}$, and it eventually collapsed to a black hole surrounded by a disk of mass $0.1-0.2 M_{\odot}$, which might be the central engine of a gamma-ray burst associated with GW170817 [7,8]. At the merger and during the postmerger stage, ejection of matter with mass $\sim 0.05 M_{\odot}$ occurred. At the merger, a neutron-rich material, which can synthesize heavy $r$-process elements, was ejected in the dynamical process, while in 
the postmerger stage, the mass ejection occurred from a disk (or torus) surrounding the remnant hypermassive neutron star and the black hole subsequently formed. The observational results suggest that the neutron richness of the postmerger ejecta is unlikely to be very high. A canonical interpretation for this is that the neutrino irradiation from the hypermassive neutron star to the ejecta is strong enough to reduce the neutron richness. However, physically wellmodeled and well-resolved numerical simulations taking into account full general relativity, neutrino transport, and angular momentum transport by magnetohydrodynamics or viscous hydrodynamics effects have not been performed yet. Thus, it is not clear whether the canonical interpretation is really correct.

Although the electromagnetic observation for GW170817 provides us with rich information for the neutron-star merger, it will not be always the case that the next events have similar features for the electromagnetic counterparts, as the GW190425 event suggests [9]. For GW170817, the total mass of two neutron stars is not so large that the remnant could form a massive neutron star at least temporarily. On the other hand, for more massive binary neutron stars or for the case of black hole-neutron star binaries, a black hole surrounded by a disk is the expected remnant. For this case, neutrino emission as strong as that from hypermassive neutron stars is absent, and the properties of the postmerger ejecta can be different from those for GW170817 [10-21]. It is worthy to explore in detail the ejecta properties for the case that a black hole is the immediate remnant of neutronstar mergers.

There is a lot of previous work exploring the ejecta from the system composed of a black hole and a disk surrounding it. However, the previous work has been performed with some simplifications. In the pioneer simulation work by Fernández, Metzger, and their collaborators [10,11,13], they only qualitatively took into account the general relativistic effects and the radiation transfer effects of neutrinos. In particular, the spacetime structure around the black hole was only qualitatively considered. In Refs. $[12,14]$, the authors took into account the neutrino irradiation effect carefully, but again they only qualitatively - if at all — took into account the general relativistic effects and black-hole spacetime. In Refs. [15-19], the authors performed a magnetohydrodynamics simulation taking into account the general relativistic effect with a fixed Kerr black hole as the background spacetime. However, in Refs. [15-18], the neutrino transfer effect was only approximately taken into account, and/or the equation of state employed was an approximate one (e.g., the effects of heavy nuclei are not incorporated, and in some works, the degenerate pressure of electrons, which is the key in the dense disk, is not taken into account). In Ref. [19], numerical simulations are performed only for a short term, $\sim 0.13$ s (i.e., an optimistic magnetic field for enhancing the mass ejection is initially prepared), although general relativity (background spacetime of a black hole), a realistic equation of state, and a detailed radiation transport are incorporated together for the first time.

In the present work, we perform a long-term fully general relativistic viscous neutrino-radiation hydrodynamics simulation for black hole-disk systems, approximately taking into account the neutrino irradiation effect. By focusing on the viscous evolution process, this work can provide a complementary aspect for the evolution of blackhole accretion disks to the magnetohydrodynamics work. In particular, we carefully resolve the black-hole spacetime and the inner part of the disk, and we take into account the effect of the self-gravity of the disk and the black-hole evolution by matter accretion self-consistently. Particular emphasis is put on the point that we resolve the vicinity of the black hole, including the inner region of the disk, with resolution higher than those in the previous simulations. This setting enables us to follow accurate viscous evolution of the disk.

The paper is organized as follows: In Sec. II, we briefly summarize the basic equations employed in the present simulation study, and then we describe the method to prepare the initial condition composed of the equilibrium state of disks. The dependence of the properties of the disk surrounding a black hole on the equation of state and velocity profile is also presented. Section III presents numerical results for the simulations, focusing on the properties of the ejecta and nucleosynthesis in the matter ejected from the disks. Section IV is devoted to a summary. Throughout this paper, $G, c$, and $k$ denote the gravitational constant, speed of light, and Boltzmann's constant, respectively.

\section{METHODS OF NUMERICAL COMPUTATION AND INITIAL CONDITION}

\section{A. Basic equations}

We evolve black hole-disk systems in the framework of full general relativity. For the case that the disk mass is much smaller than the black-hole mass, we are allowed to perform a simulation in a fixed background of black-hole spacetime. However, for the case that the disk mass is a substantial fraction of the black-hole mass, such assumptions may break down. In the last decade, the numerical relativity community has established methods to evolve systems composed of black holes for a long timescale stably and accurately (e.g., Ref. [22] for a review). Thus, we do not have to assume the fixed background. In the present work, we numerically solve both Einstein's equation and matter-field equations self-consistently.

The major purpose of this paper is to clarify the viscous evolution of a system composed of a self-gravitating disk surrounding a spinning low-mass black hole, which is the plausible outcome formed after the merger of a low-mass black hole-neutron star binary or a binary neutron star with high total mass. For the disk evolution in nature, the 
TABLE I. Initial conditions for the numerical simulation. Described are the model name, black-hole mass, disk mass, black-hole dimensionless spin, radii at the inner and outer edges of the disk $\left(r_{\text {in }}\right.$ and $\left.r_{\text {out }}\right)$, entropy per baryon $(s / k), n$, electron fraction $\left(Y_{e}\right)$ for the disk, and $\alpha_{\nu} H$. The units of the mass are $M_{\odot}$, and the units of $r_{\text {in }}$ and $r_{\text {out }}$ are $G M_{\mathrm{BH}} / c^{2} \approx 4.43 \mathrm{~km}$. The last column shows whether the neutrino irradiation is switched on or off.

\begin{tabular}{|c|c|c|c|c|c|c|c|c|c|c|}
\hline Model & $M_{\mathrm{BH}}$ & $M_{\text {disk }}$ & $\chi$ & $r_{\text {in }}$ & $r_{\text {out }}$ & $s / k$ & $n$ & $Y_{e}$ & $\alpha_{\nu} H(\mathrm{~km})$ & $\begin{array}{l}\text { Neutrino } \\
\text { irradiation }\end{array}$ \\
\hline K8 & 3.0 & 0.10 & 0.8 & 2.0 & 41 & 6 & $1 / 7$ & $0.07-0.5$ & 0.45 & yes \\
\hline K8h & 3.0 & 0.10 & 0.8 & 2.0 & 41 & 6 & $1 / 7$ & $0.07-0.5$ & 0.90 & yes \\
\hline $\mathrm{K} 8 \mathrm{~s}$ & 3.0 & 0.10 & 0.8 & 2.0 & 41 & 6 & $1 / 7$ & $0.07-0.5$ & 1.35 & yes \\
\hline K8n & 3.0 & 0.10 & 0.8 & 2.0 & 41 & 6 & $1 / 7$ & $0.07-0.5$ & 0.45 & no \\
\hline D8 & 3.0 & 0.10 & 0.8 & 2.0 & 29 & 6 & $1 / 10$ & $0.07-0.5$ & 0.45 & yes \\
\hline Y8 & 3.0 & 0.10 & 0.8 & 2.0 & 41 & 8 & $1 / 8$ & 0.1 & 0.45 & yes \\
\hline K6 & 3.0 & 0.10 & 0.6 & 2.8 & 41 & 6 & $1 / 10$ & $0.07-0.5$ & 0.45 & yes \\
\hline $\mathrm{C} 8$ & 3.0 & 0.50 & 0.8 & 2.0 & 58 & 6 & $1 / 7$ & $0.07-0.5$ & 0.45 & yes \\
\hline E8 & 3.0 & 0.03 & 0.8 & 2.0 & 29 & 6 & $1 / 7$ & $0.07-0.5$ & 0.45 & yes \\
\hline
\end{tabular}

magnetohydrodynamics or viscous heating/angular momentum transport is one of the key processes. In addition, the neutrino cooling of the disk and neutrino irradiation to the matter are key processes for the disk evolution and for determining the property of the matter ejected from the system. In this work, we choose basic equations as follows: Einstein's equation, the viscous hydrodynamics equations, the evolution equation for the viscous tensor, the evolution equations for the lepton fractions including the electron fraction, and neutrino-radiation transfer equations. Here, for the equations for the lepton fractions, we take into account electron and positron capture, electron-positron pair annihilation, nucleon-nucleon bremsstrahlung, and plasmon decay [20]. For solving Einstein's equation, we employ the Baumgarte-Shapiro-Shibata-Nakamura formalism [23] together with the puncture formulation [24], Z4c constraint propagation prescription [25], and fifth-order Kreiss-Oliger dissipation. In reality, the viscous angular momentum transport is likely to be induced effectively by a magnetohydrodynamics process [26]. In the present work, this process is approximated by viscous hydrodynamics. All these basic equations are the same as those in Ref. [20], in which we performed a simulation for the system of a massive neutron star and disk.

One crucial difference of the present simulation from the previous ones $[20,21]$ is that we have to evolve a spinning black hole located at the center stably and accurately for a long timescale, at least $\sim 3-5 \mathrm{~s}$. This can be achieved only by employing a very high grid resolution around the central region. We find that to follow the black hole with a dimensionless spin of $\chi=0.8$ accurately-i.e., to get the evolution of mass and spin for the black hole with reasonable accuracy - the grid spacing in the central region, $\Delta x_{0}$, should be smaller than $\sim 0.02 G M_{\mathrm{BH}} / c^{2}$ (see Sec. III), where $M_{\mathrm{BH}}$ is the initial mass of the black hole: For $M_{\mathrm{BH}}=3 M_{\odot}$, the grid spacing has to be smaller than $\sim 80 \mathrm{~m}$. Otherwise, the area and dimensionless spin of the black hole, respectively, increase and decrease spuriously and significantly. In the present work, we employ $\Delta x_{0}=0.016 G M_{\mathrm{BH}} / c^{2}$. We also perform simulations with $\Delta x_{0}=0.0133 G M_{\mathrm{BH}} / c^{2}$ and $0.020 G M_{\mathrm{BH}} / c^{2}$ for a particular model (referred to as the K8 model in Table I) and show the convergence property in Sec. III F. We note that such a high resolution helps in accurately resolving the inner region of the disk.

As mentioned above, a sufficiently high grid resolution is the key for a reliable simulation. Furthermore, we have to evolve the disk for the timescale of $\gtrsim 2 \times 10^{5} G M_{\mathrm{BH}} /$ $c^{3} \sim 3 \mathrm{~s}$, because the viscous timescale of the disk is much longer than the dynamical timescale (the typical orbital period) of the system (cf. Sec. II D). With these requirements (high-resolution and long-term simulation is required), however, a three-dimensional simulation under no assumption of symmetry is still quite expensive. Thus, as in Refs. [20,21], we assume the axial symmetry of the spacetime as well as the reflection symmetry with respect to the equatorial plane: We employ a cartoon method $[27,28]$ to impose the symmetry for solving Einstein's equation; i.e., we employ the Cartesian coordinates $(x, y, z)$ and evolve the system only in the $(x, z)$ plane (the $y=0$ plane).

As already mentioned, in addition to Einstein's equation, we solve the viscous hydrodynamics equation, evolution equations for the viscous tensor and lepton fractions, and the radiation transfer equation. These equations are solved in the cylindrical coordinates composed of $(x, z)$ (see Ref. [29] for basic equations of viscous hydrodynamics).

For the $x$ and $z$ directions, the following nonuniform grid is used for the present numerical simulation: For $x \leq x_{0}=0.8 G M_{\mathrm{BH}} / c^{2}$, a uniform grid is used, and for $x>x_{0}$, the grid spacing $\Delta x$ is increased uniformly as $\Delta x_{i+1}=1.01 \Delta x_{i}$, where the subscript $i$ denotes the $i$ th grid with $x=0$ at $i=0$. For $z$, the same grid structure as for $x$ is used. The black-hole horizon is always located in the uniform grid zone. The location of the outer boundaries 
along each axis is chosen to be $\approx 1400 G M_{\mathrm{BH}} / c^{2} \approx$ $6100\left(M_{\mathrm{BH}} / 3 M_{\odot}\right) \mathrm{km}$ in this study.

\section{B. Initial condition}

We prepare an axisymmetric equilibrium state for the black hole-disk system in the framework of full general relativity as the initial condition for our numerical simulation. For the gravitational field equations, we employ a puncture formulation developed in Ref. [30] with the following line element:

$$
\begin{aligned}
d s^{2}= & -\alpha^{2} c^{2} d t^{2}+\psi^{4}\left[e^{2 \eta}\left(d r^{2}+r^{2} d \theta^{2}\right)\right. \\
& \left.+r^{2} \sin ^{2} \theta\left(\beta^{\varphi} d t+d \varphi\right)^{2}\right] .
\end{aligned}
$$

Here, we employ spherical polar coordinates, and $\alpha, \psi, \beta^{\varphi}$, and $\eta$ are functions of $r$ and $\theta$. Note that $\eta$ and $\beta$ vanish for the nonspinning black hole. In the following, we denote the dimensionless spin parameter by $\chi$, which is chosen to be 0.8 or 0.6 .

We consider the case that the fluid four-velocity has the form of $u^{r}=0=u^{\theta}$ and $u^{\varphi}=\Omega u^{t}$, and the fluid is isentropic. Here $\Omega$ is the angular velocity, which is also a function of $r$ and $\theta$. Then, under the assumption of the isentropy, the Euler equation is integrated to give the first integral in the form

$$
\frac{h}{u^{t}}+\frac{1}{c^{2}} \int h u_{\varphi} d \Omega=C,
$$

where $h$ is the specific enthalpy and $C$ is a constant. Using the rest-mass density $\rho$, the specific internal energy $\varepsilon$, and the pressure $P, h$ is written as $c^{2}+\varepsilon+P / \rho$. Using the normalization relation for $u^{\mu}, u^{\mu} u_{\mu}=-c^{2}, u^{t}$ is written as

$$
u^{t}=\left(\alpha^{2}-c^{-2} \gamma_{\varphi \varphi}\left(\Omega+\beta^{\varphi}\right)^{2}\right)^{-1 / 2},
$$

where $\gamma_{\varphi \varphi}=\psi^{4} r^{2} \sin ^{2} \theta$.

In this paper, we simply assume that $j=c^{-2} h u_{\varphi}$ is a function of $\Omega$ in the form of

$$
j=A_{n} \Omega^{-n},
$$

where $A_{n}$ and $n$ are constants (see Ref. [31] for a more careful choice). In the Newtonian limit, $j \approx u_{\varphi} \approx \varpi^{2} \Omega$, with $\varpi=r \sin \theta$, and hence $\Omega \propto \varpi^{-2 /(n+1)}$. Thus, for $n=1 / 3$, the Keplerian angular velocity is recovered in the Newtonian limit, and for $n=0$, the specific angular momentum is constant. Since the angular velocity profile of the remnant disk of neutron-star mergers is close to the Keplerian, we should try to employ a value of $n$ as close as $1 / 3$. We find that the disk mass in the equilibrium states with $1 / 6 \leq n<1 / 3$ in our present setting becomes quite small if the radius of the outer edge of the disk is a reasonable value for a remnant of neutron-star mergers as $\lesssim 50 G M_{\mathrm{BH}} / c^{2}$ (see discussion below). Thus, we employ the values of $n \leq 1 / 7$.

For a given value of $n$ and an equation of state, we have to determine $\Omega, h, A_{n}$, and $C$. In the following, we describe the basic equations for this procedure. First, Eq. (2.4) is rewritten as

$$
\Omega^{n}\left(\Omega+\beta^{\varphi}\right) h u^{t} \gamma_{\varphi \varphi}=A_{n} c^{2}
$$

where we use

$$
u_{\varphi}=u^{t}\left(\Omega+\beta^{\varphi}\right) \gamma_{\varphi \varphi} .
$$

Using Eq. (2.4), Eq. (2.2) is rewritten as

$$
\frac{h}{u^{t}}+\frac{A_{n} \Omega^{1-n}}{1-n}=h\left(\frac{1}{u^{t}}+\frac{u_{\varphi} \Omega}{c^{2}(1-n)}\right)=C .
$$

From Eqs. (2.3) and (2.5), we also obtain

$\frac{A_{n} \Omega^{-n}\left[c^{2} \alpha^{2}-\gamma_{\varphi \varphi}\left(\Omega+\beta^{\varphi}\right)^{2}\right]}{\gamma_{\varphi \varphi}\left(\Omega+\beta^{\varphi}\right)}=\left(C-\frac{A_{n} \Omega^{1-n}}{1-n}\right)$.

This algebraic equation is used to determine $\Omega$ for given values of $A_{n}$ and $C$ with gravitational fields computed.

$A_{n}$ and $C$ are determined by choosing the inner and outer edges of the disk in the equatorial plane. Since the values of $h$ are identical at such edges, Eq. (2.7) gives

$$
\left(\frac{1}{u^{t}}+\frac{u_{\varphi} \Omega}{c^{2}(1-n)}\right)_{\text {in }}=\left(\frac{1}{u^{t}}+\frac{u_{\varphi} \Omega}{c^{2}(1-n)}\right)_{\text {out }},
$$

where "in" and "out" in the subscripts indicate the quantities at the inner and outer edges, respectively. Here, $u^{t}$ and $u_{\varphi}$ are given by Eqs. (2.3) and (2.6), respectively.

In addition, Eq. (2.4) is written to $\left(j \Omega^{n}\right)_{\text {in }}=\left(j \Omega^{n}\right)_{\text {out }}$, which leads to

$$
\left(u_{\varphi} \Omega^{n}\right)_{\text {in }}=\left(u_{\varphi} \Omega^{n}\right)_{\text {out }} .
$$

Equations (2.9) and (2.10) constitute simultaneous equations for $\Omega_{\text {in }}$ and $\Omega_{\text {out }}$, and hence, by solving these equations, we first determine these angular velocities. Subsequently, using Eqs. (2.5) and (2.7), $A_{n}$ and $C$ are determined for the value of $h$ at $\rho=\rho_{\min }$, where the minimum values of $h$ and $\rho_{\min }$ are found for a given tabulated equation of state. Then, $\Omega$ at each point is determined by solving Eq. (2.8). Once $A_{n}, C$, and $\Omega$ at each point are determined, $h$ is determined from Eq. (2.7).

For a given value of $h$, thermodynamical variables $\rho, \varepsilon$, and $P$ are determined through the equation of state given. In this work, we employ a tabulated equation of state based on the DD2 equation of state [32] for a relatively high-density part and the Timmes equation of state for a low-density part [33]. We choose the lowest rest-mass density to be 

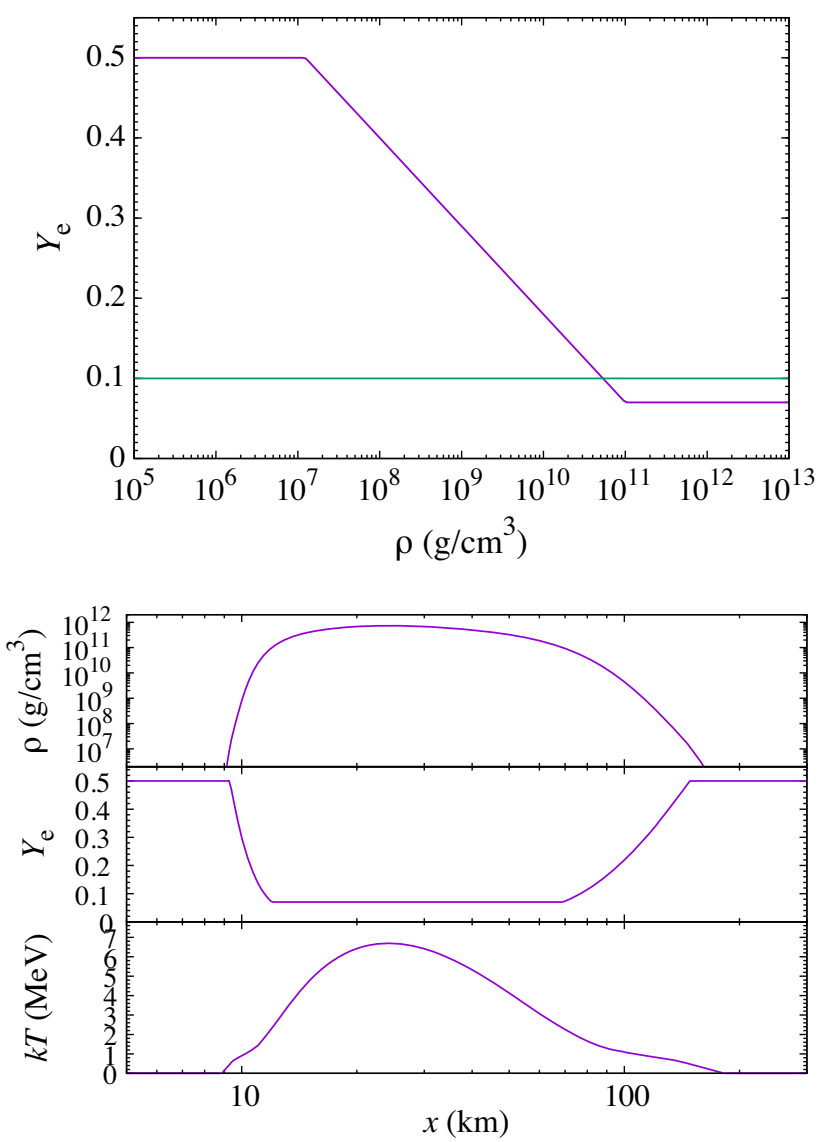

FIG. 1. Top: Two relations for $Y_{e}$ employed for constructing equilibrium states of disks surrounding the black hole. Bottom: Density, electron fraction, and temperature $(k T)$ as functions of the radius in the equatorial plane for a disk model (model K8 in Table I).

$0.1 \mathrm{~g} / \mathrm{cm}^{3}$ in the table. In this equation of state, $\varepsilon, P$, and $h$ are functions of $\rho, Y_{e}$, and $T$, where $Y_{e}$ and $T$ are the electron fraction and matter temperature, respectively. Thus, to determine $\rho, Y_{e}$, and $T$ from $h$, we need to employ two conditions for relating these three variables. One condition is just the assumption that the specific entropy $s$ is constant because Eq. (2.2) is derived under this condition. For the other, we adopt two relations. In one case, we assume a relation between $Y_{e}$ and $\rho$ in the form $\rho\left(Y_{e}\right)$ (see Fig. 1), and in the other case, we simply set $Y_{e}=0.1$. For the former case, we derive an approximate relation of $\rho\left(Y_{e}\right)$ from our numerical results for the remnant of binary neutron-star mergers [34] (see also, e.g., Refs. [35-37]), for which the value of $Y_{e}$ in the major part of the disk is approximately determined by weak interaction processes. For this model, the value of $Y_{e}$ is larger for the smaller density, because the effect of the electron degeneracy, compared to the thermal effects, becomes weaker. We basically employ this as the fiducial model, and the equation of state is simply referred to by specifying the value of $s / k$. The equation of state with $Y_{e}=0.1$ could be an approximate model for the remnant of black hole-neutron star mergers [38,39]. When we refer to this model, we always mention $Y_{e}=0.1$.

The bottom panel of Fig. 1 displays the rest-mass density, electron fraction, and temperature as functions of the radius in the equatorial plane for a typical equilibrium model employed in this paper as an initial condition for the simulation (model $\mathrm{K} 8$ in Table I). For this model, $M_{\mathrm{BH}}=3 M_{\odot}, \chi=0.8, M_{\text {disk }}=0.1 M_{\odot}, \quad r_{\text {in }}=$ $2.0 G M_{\mathrm{BH}} / c^{2}, r_{\text {out }} \approx 41 G M_{\mathrm{BH}} / c^{2}, s / k=6$, and $n=1 / 7$. We find that the maximum density is $\lesssim 10^{12} \mathrm{~g} / \mathrm{cm}^{3}$, and the maximum temperature (in terms of $k T$ ) is $\sim 7 \mathrm{MeV}$. In the dense region, the electron degeneracy is high, and as a result, the electron fraction is low, $\leq 0.1$. This is a typical structure of the disk around low-mass black holes, with $M_{\text {disk }}=O\left(0.1 M_{\odot}\right)$.

Figure 2 displays several relations for the radius of the outer edge, $r_{\text {out }}$, and baryon mass, $M_{\text {disk }}$, of the disk for a variety of the values of $n$ and $s / k$. In this plot, we fix $M_{\mathrm{BH}}=3 M_{\odot}, \chi=0.8$, and $r_{\text {in }}=2.0 G M_{\mathrm{BH}} / c^{2}$. On the other hand, we employ a variety of the equations of state with $s / k=6-8$ and a wide range of $n$. The left panel shows the outer edge of the disk, $r_{\text {out }}$, as a function of $n$ for $M_{\text {disk }}=0.1 M_{\odot}$. For the larger values of $n$ toward $1 / 3$, the velocity profile of the disk approaches the Keplerian profile and the disk becomes geometrically thin. As a result, to preserve a given value of $M_{\text {disk }}$, the extent of the disk (i.e., $r_{\text {out }}$ ) needs to be increased with $n$. It is also found that for higher values of $s / k$, the extent of the disk has to be larger. The reason for this is that for higher values of $s / k$, the pressure for a given value of the density is larger, and hence the overall density becomes relatively small.

The right panel of Fig. 2 shows $M_{\text {disk }} / M_{\mathrm{BH}}$ as a function of $n$ for $r_{\mathrm{out}}=40 G M_{\mathrm{BH}} / c^{2}$. This clarifies that for larger values of $n$, the disk mass is smaller for the given extent of the disk, and for smaller values of $s / k$, the disk mass is larger. It is also found that for the $Y_{e}=0.1$ case, the disk mass is larger for a given value of $s / k$. This is reasonable because for smaller values of $Y_{e}$, the electron degeneracy pressure is less important, and hence, to enhance the pressure, a higher value of the rest-mass density is needed.

In Fig. 3, we compare the results for $\chi=0.6$ and 0.8. For $\chi=0.6$, the inner edge of the disk cannot be as small as that for $\chi=0.8$, because the location of the innermost stable circular orbit around the black hole is closer to the black hole for the larger value of $\chi$ for a given black-hole mass. Here we set $r_{\mathrm{in}}=2.8 G M_{\mathrm{BH}} / c^{2}$ for $\chi=0.6$, while it is $2.0 G M_{\mathrm{BH}} / c^{2}$ for $\chi=0.8$. It is found for $\chi=0.6$ that the value of $r_{\text {out }}$ becomes larger for given parameters of $s, n$, and $M_{\text {disk }}$ than for $\chi=0.8$. It is also found that the disk mass is smaller for the given values of $s, n$, and $r_{\text {out }}=$ $40 G M_{\mathrm{BH}} / c^{2}$ than for $\chi=0.8$. The reason for these results is that for smaller values of $\chi$, the gravitational potential of the black hole is shallower, and hence the amount of the bounded material becomes smaller. Thus, to obtain a model with the same disk mass and the same value of 

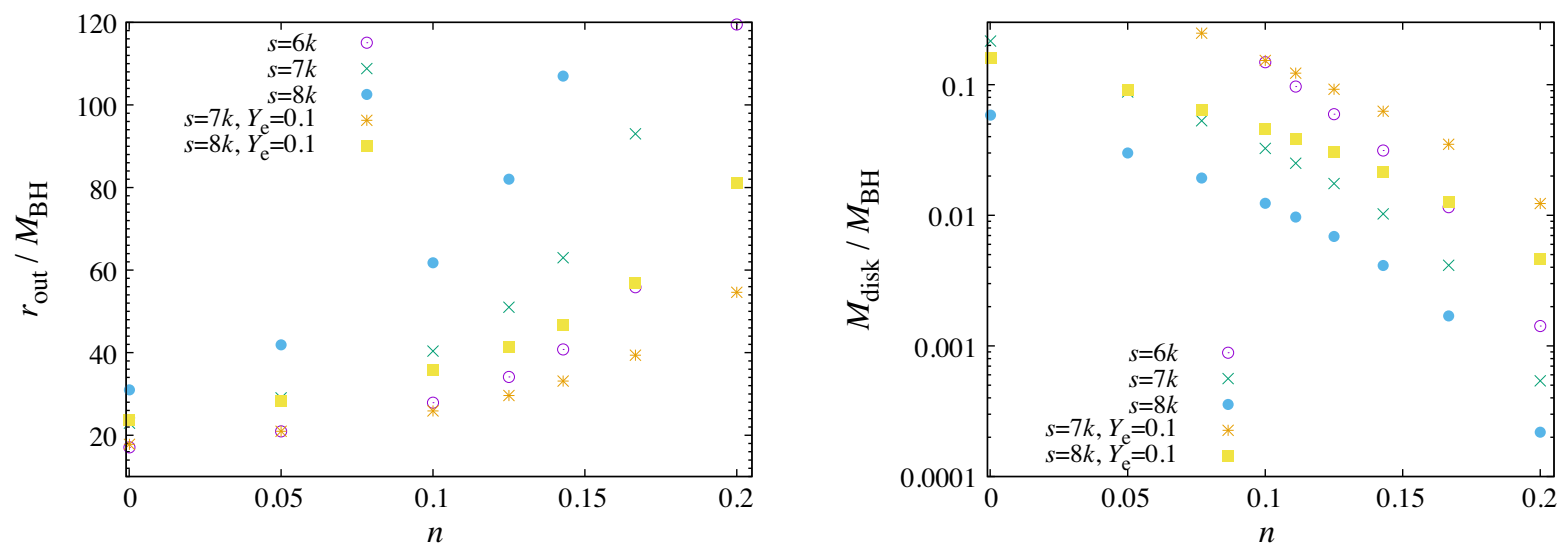

FIG. 2. Left: $r_{\text {out }} /\left(G M_{\mathrm{BH}} / c^{2}\right)$ as a function of $n$ for the case of $M_{\text {disk }} / M_{\mathrm{BH}}=1 / 30$. Right: $M_{\text {disk }} / M_{\mathrm{BH}}$ as a function of $n$ for $r_{\text {out }}=40 G M_{\mathrm{BH}} / c^{2}$. For both panels, $r_{\mathrm{in}}=2.0 G M_{\mathrm{BH}} / c^{2}$ and $\chi=0.8$. The equilibrium states with $n=1 / 10-1 / 7, s / k=6$ or 8 , and $r_{\text {out }} / M_{\mathrm{BH}}=29-58$ are employed as initial conditions for numerical simulation (cf. Table I).
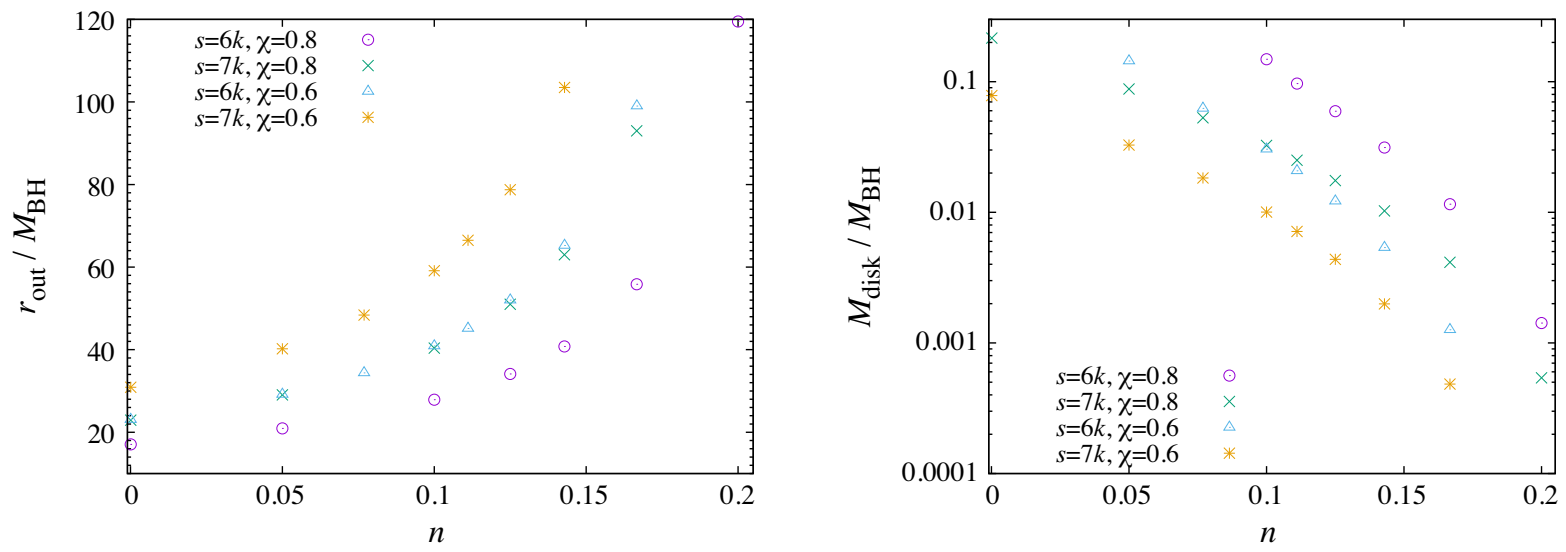

FIG. 3. The same as Fig. 2, but for the comparison between $\chi=0.8$ and 0.6. For $\chi=0.8$ and $0.6, r_{\text {in }}=2.0 G M_{\mathrm{BH}} / c^{2}$ and $2.8 G M_{\mathrm{BH}} / c^{2}$, respectively (see Table I for the equilibrium states used for numerical simulation).

$r_{\text {out }} /\left(G M_{\mathrm{BH}} / c^{2}\right)$ for $\chi=0.6$ as for $\chi=0.8$, we need to prepare a smaller value of $s$ or a smaller value of $n$.

The simulations are performed for several models as the initial conditions. First, we employ models of $s / k=6$ and $n=1 / 7$ for the fiducial $Y_{e}$ case, and $s / k=8$ and $n=1 / 8$ for the $Y_{e}=0.1$ case, with $\chi=0.8$ (see models $\mathrm{K} 8$ and Y8 in Table I). For both cases, we set $\left(r_{\text {in }}, r_{\text {out }}\right) \approx\left(2 G M_{\mathrm{BH}} / c^{2}\right.$, $\left.41 G M_{\mathrm{BH}} / c^{2}\right)$, and we set the mass of the black hole and the baryon rest mass of the disk to be $M_{\mathrm{BH}}=3 M_{\odot}$ and $M_{\text {disk }}=0.1 M_{\odot}$, respectively. We also employ a compact disk model (D8) for which $s / k=6, n=1 / 10$, and $\left(r_{\text {in }}, r_{\text {out }}\right) \approx\left(2 G M_{\mathrm{BH}} / c^{2}, 29 G M_{\mathrm{BH}} / c^{2}\right)$, with the same mass and spin- $M_{\mathrm{BH}}=3 M_{\odot}, \chi=0.8$, and $M_{\text {disk }}=$ $0.1 M_{\odot}$-as those for models $\mathrm{K} 8$ and Y8. We will show that the slight difference in $s$ and $n$ does not drastically change the overall dynamics of the disk or the properties of the ejecta, although for initially more compact disks, the ejecta mass becomes smaller as a natural consequence. In addition, we employ one model with $\chi=0.6$, $M_{\mathrm{BH}}=3 M_{\odot}$, and $M_{\mathrm{disk}}=0.1 M_{\odot}$ in which $n=1 / 10$, $s=6 k$, and $\left(r_{\text {in }}, r_{\text {out }}\right) \approx\left(2.8 G M_{\mathrm{BH}} / c^{2}, 41 G M_{\mathrm{BH}} / c^{2}\right)$ to explore the dependence of the numerical results on the black-hole spin.

We note that the outer edge of the disk should not be very large for modeling the merger remnant of neutron-star binaries; numerical-relativity simulations have shown that $r_{\text {out }}$ is between 100 and $200 \mathrm{~km}$. For this reason, we fiducially set $r_{\text {out }} \approx 40 G M_{\mathrm{BH}} / c^{2}$, which is $\approx 180 \mathrm{~km}$ for $M_{\mathrm{BH}}=3 M_{\odot}$. For the compact model (D8), $r_{\text {out }} \approx$ $130 \mathrm{~km}$, which is also a reasonable value.

In addition to these models, we employ initial conditions with $M_{\text {disk }}=0.5 M_{\odot}$ and $r_{\text {out }} \approx 58 G M_{\mathrm{BH}} / c^{2}$, and with $M_{\text {disk }}=0.03 M_{\odot}$ and $r_{\text {out }} \approx 29 G M_{\mathrm{BH}} / c^{2}$. For both models, we employ the fiducial $Y_{e}$ equation of state, with $\chi=0.8, M_{\mathrm{BH}}=3 M_{\odot}, r_{\mathrm{BH}}=2 G M_{\mathrm{BH}} / c^{2}, n=1 / 7$, and $s=6 k$, which are the same as those for model K8 (see Table I). By performing simulations for these initial conditions, we examine the effect of the mass (i.e., the effects by the density and temperature) of the disk on the evolution of the system. We note that the initial condition with $M_{\text {disk }}=0.03 M_{\odot}$ is a good model for neutron-star mergers, but that with $0.5 M_{\odot}$ is not. Rather, such a high- 
mass disk may be a good model for the remnant of the massive stellar core collapse to a black hole.

Because the mass ratio, $M_{\text {disk }} / M_{\mathrm{BH}}$, of the initial conditions employed here is fairly large $(1 / 100-1 / 6)$, and hence the disks are weakly self-gravitating, they may be subject to nonaxisymmetric deformation even if the angular velocity profile is close to the Keplerian. Previous work in general relativity (e.g., Refs. [40-42]) shows that if the self-gravity of the disks is not extremely large, spiral arms are formed and contribute to angular momentum transport by the gravitational torque exerted by the nonaxisymmetric structure. In our simulation, such nonaxisymmetric effects cannot be taken into account, but the angular momentum transport is incorporated through the viscous hydrodynamics. Moreover, the previous work [40-42] shows that the density enhancement in the spiral arms is not very strong, and the angular momentum transport is much less efficient than that in the viscous hydrodynamics with the alpha viscous parameter [43], $\alpha_{\nu}=O\left(10^{-2}\right)$. Therefore, we suppose that the nonaxisymmetric deformation effects would not be very important for the models that we employ here.

\section{Method for analyzing ejecta}

Here, we briefly summarize how we identify the matter as ejecta. The unbound matter should be considered as ejecta. In this work, we employ the following condition for identifying matter in an unbound orbit: $\left|h u_{t}\right|>h_{\min } c^{2}$, where $h_{\min }$ denotes the minimum value of the specific enthalpy $h$ in the chosen tabulated equation of state, which is $\approx 0.9987 c^{2}$, and $u_{t}$ is a negative quantity. The reason why $h_{\min }$ is smaller than $c^{2}$ is that the effect of the binding energy of the nucleus is present in the equation of state.

To analyze the ejecta, we first extract the outgoing component of the matter at the radius of $r_{\mathrm{ext}}=$ $2000-4000 \mathrm{~km}$ and search for the ejecta component. Here, by changing the extraction radius, we examine the convergence of the ejecta mass. In addition, we analyze the matter located within a sphere of $r=r_{\text {ext }}$, and the component with $\left|h u_{t}\right|>h_{\min } c^{2}$ is identified as the ejecta. By summing up these two components, we determine the quantities of the ejecta.

For the ejecta component escaping from a sphere of $r=r_{\text {ext }}$, we define the ejection rates of the rest mass and total energy at a given radius by

$$
\begin{gathered}
\dot{M}_{\mathrm{eje}}:=c^{-1} \oint \rho \sqrt{-g} u^{i} d S_{i}, \\
\dot{E}_{\mathrm{eje}}:=c^{-1} \oint \rho \hat{e} \sqrt{-g} u^{i} d S_{i},
\end{gathered}
$$

where $g$ denotes the determinant of the spacetime metric and $\hat{e}:=h \alpha u^{t}-P /\left(\rho \alpha u^{t}\right)$. The surface integral is performed at $r=r_{\text {ext }}$ with $d S_{i}=\delta_{i r} r_{\mathrm{ext}}^{2} d \theta d \varphi$ for the ejecta component. Here, we note that $\rho \sqrt{-g} u^{t}$ obeys the continuity equation of the rest-mass density, and $\rho \hat{e} \sqrt{-g} u^{t}$ obeys the energy conservation equations in the absence of gravity. Hence, for the region far from the central object, the time integration of these quantities is conserved. Thus, by performing the time integration, the total rest mass and energy of the ejecta (which escape away from a sphere of $r=r_{\text {ext }}$ ) are calculated by

$$
\begin{aligned}
M_{\text {eje,esc }}(t) & :=\int{ }^{t} \dot{M}_{\text {eje }} d t, \\
E_{\text {eje,esc }}(t) & :=\int{ }^{t} \dot{E}_{\text {eje }} d t .
\end{aligned}
$$

In addition, we add the rest mass for the ejecta component located inside a sphere of $r=r_{\mathrm{ext}}, M_{\mathrm{eje}, \mathrm{in}}(t)$, giving the total ejecta mass, $M_{\text {eje }}=M_{\text {eje,esc }}+M_{\text {eje,in }}$.

We note that far from the central object, $E_{\text {eje,esc }}$ is approximated by

$E_{\text {eje,esc }} \approx M_{\text {eje,esc }} c^{2}+U+T_{\text {kin }}+\frac{G M_{\mathrm{BH}} M_{\text {eje,esc }}}{r_{\text {ext }}}$,

where $U$ and $T_{\text {kin }}$ are the values of the internal energy and kinetic energy of the ejecta at $r_{\text {ext }} \rightarrow \infty$, respectively. The last term of Eq. (2.15) approximately denotes the contribution of the potential binding energy to the matter at $r=r_{\text {ext }}$. This correction is necessary because the kinetic energy and internal energy of the matter are decreased during the motion from $r=r_{\text {ext }}$ to infinity, and the decrease cannot be neglected for $r_{\mathrm{ext}} \lesssim 10^{3} G M_{\mathrm{BH}} / c^{2} \approx 4500 \mathrm{~km}$, which can modify the ejecta velocity $v_{\text {eje }}$ by the order of $10^{-2} \mathrm{c}$. Since the ratio of the internal energy to the kinetic energy of the ejecta decreases with its expansion, we may approximate $U / T_{\text {kin }} \approx 0$, and hence, $E_{\text {eje,esc }}$ by $E_{\text {eje,esc }} \approx$ $M_{\text {eje,esc }} c^{2}+T_{\text {kin }}+G M_{\mathrm{BH}} M_{\text {eje,esc }} / r_{\text {ext }}$ for the region far from the central object. We then define the average velocity of the ejecta (for the component that escapes from a sphere of $r=r_{\mathrm{ext}}$ ) by

$v_{\mathrm{eje}}:=\sqrt{\frac{2\left(E_{\mathrm{eje}, \mathrm{esc}}-M_{\mathrm{eje}, \mathrm{esc}} c^{2}-G M_{\mathrm{BH}} M_{\mathrm{eje}, \mathrm{esc}} / r_{\mathrm{ext}}\right)}{M_{\mathrm{eje}, \mathrm{esc}}}}$.

We note that the correction of the gravitational potential energy $G M_{\mathrm{BH}} M_{\text {eje,esc }} / r_{\text {ext }}$ is important for $r_{\text {ext }} \lesssim 10^{4} \mathrm{~km}$, and just by taking into account this correction, the values of $v_{\text {eje }}$ become only weakly dependent on the extraction radius, $r_{\text {ext }}$.

\section{Viscous parameter}

For the viscous hydrodynamics, we need to input the viscous coefficient $\nu$. In this work, we set $\nu=\alpha_{\nu} h c_{s} H / c^{2}$, 
where $\alpha_{\nu}$ is the dimensionless viscous coefficient (the socalled alpha parameter), $c_{s}$ is the sound velocity, and $H$ is a scale height. We basically employ $\alpha_{\nu}=0.05$, taking into account the results of recent magnetohydrodynamics simulations of Refs. $[16,18]$, which indicate that the magnitude of the effective viscous parameter is high, with $\alpha_{\nu} \approx$ $0.05-0.1$ in the vicinity of spinning black holes.

For the fiducial model, we set $H=9 \mathrm{~km} \approx 2 G M_{\mathrm{BH}} / c^{2}$. That is, we set it approximately equal to the radius at the innermost stable circular orbit around the Kerr black hole of $\chi=0.8$. For the outer part of the disk, the value of the scale height might be larger than $H \approx 9 \mathrm{~km}$, because it could be approximately $c_{s} / \Omega$ in the standard accretion disk theory [43]. However, for a nonstationary system, this is not likely to be the case, if we suppose that the viscosity is effectively enhanced by the turbulence caused by the magnetorotational instability (MRI) [26], because the exponential growth rate of the MRI is proportional to the local angular velocity, $\Omega$ (for the Keplerian angular velocity profile, the growth rate is $3 \Omega / 4$ ). That is, to establish the turbulence by enhancing the magnetic-field strength by several orders of magnitude until the saturation of the magnetic-field growth, it takes a timescale of $\gtrsim 10 /(3 \Omega / 4)$. Here, the rotational period of the disk is $2 \pi / \Omega \approx 310 \mathrm{~ms}$ for a radius of $r=$ $1000 \mathrm{~km}$ and a black-hole mass of $3 M_{\odot}$ assuming the Kepler motion of the disk, while the timescale of the mass ejection, which is triggered by the viscous heating in the inner part of the disk, is $\sim 0.5\left(\alpha_{\nu} / 0.05\right)^{-1}(H / 9 \mathrm{~km})^{-1} \mathrm{~s}$ (see Sec. III B). Thus, it would not be realistic to employ a large value of it for the outer part of the disk. Employing $c_{s} / \Omega$ as the scale height could result in an overestimated value of the scale height for a large value of $c_{s}$, and the mass ejection process could be induced from an outer part of the disk in an unrealistic manner for the early evolution stage of disks with $t \ll 1 \mathrm{~s}$.

To examine the dependence of the numerical results on the value of $\nu$, we change $\alpha_{\nu} H$ from $0.45 \mathrm{~km}$ to $0.90 \mathrm{~km}$ and $1.35 \mathrm{~km}$ for the model of the K8 series (see Table I). We note that in our setting of $\nu$, varying $H$ is equivalent to varying $\alpha_{\nu}$; numerical results for $\alpha_{\nu}=0.05$ and $H=9 \mathrm{~km}$ are the same as those, e.g., for $\alpha_{\nu}=0.01$ and $H=45 \mathrm{~km}$. However, for simplicity, in the following, the viscous coefficients with $\alpha_{\nu} H=0.90 \mathrm{~km}$ and $1.35 \mathrm{~km}$ are referred to as $\alpha_{\nu}=0.10$ and $\alpha_{\nu}=0.15$ with $H=9 \mathrm{~km}$, respectively.

The viscous timescale (for heating and angular momentum transport) is written approximately by

$$
\begin{aligned}
\tau_{\text {vis }}:=\frac{R^{2}}{\nu} \approx & 0.37 \mathrm{~s}\left(h c^{-2}\right)^{-1}\left(\frac{\alpha_{\nu}}{0.05}\right)^{-1}\left(\frac{c_{s}}{0.05 c}\right)^{-1} \\
& \times\left(\frac{H}{9 \mathrm{~km}}\right)^{-1}\left(\frac{R}{50 \mathrm{~km}}\right)^{2},
\end{aligned}
$$

where $R$ denotes the cylindrical radius of the disk. As we show in Sec. III B, the evolution timescale for our choice of the viscous coefficient is indeed of the order of $0.1 \mathrm{~s}$.
We note that the dynamical timescale of the disk is approximately

$\tau_{\mathrm{dyn}}:=2 \pi \sqrt{\frac{R^{3}}{G M_{\mathrm{BH}}}}=3.5 \mathrm{~ms}\left(\frac{R}{50 \mathrm{~km}}\right)^{3 / 2}\left(\frac{M}{3 M_{\odot}}\right)^{-1 / 2}$,

and hence, $\tau_{\text {vis }}$ is much longer than $\tau_{\text {dyn }}$. Thus, if the system evolves by the viscous process, the evolution should proceed in a quasistationary manner.

\section{NUMERICAL RESULTS}

\section{A. Models}

Numerical computations are performed for the black hole-disk systems summarized in the previous section (cf. Table I). For many models in this paper, the simulations are performed taking into account the neutrino irradiation effect. For one model, K8n, we switch off the neutrino irradiation effect to examine whether it is important or not. In this work, we do not incorporate a heating effect by the neutrino pair annihilation [20], because the neutrino luminosity is not very high for most of the evolution stage of the system in the models employed. Only in the very early stage with $t \lesssim 10 \mathrm{~ms}$ is the neutrino luminosity high $\left(\gtrsim 10^{53} \mathrm{erg} / \mathrm{s}\right.$ in total), and thus, the neutrino pair annihilation heating may play a role in the evolution of the disk and associated mass ejection. However, in this paper we should not consider mass ejection in such an early stage, because the system is initially in a spuriously varying phase due to a rather artificial initial condition (composed of a stationary equilibrium state of the disk of the perfect fluid), and thus, it is not very clear whether the numerical results show some physical phenomena or not for such an early stage. In one model with high disk mass (model C8: see Table I), the neutrino pair annihilation heating may be important, but we do not take it into account also for this model because model $\mathrm{C} 8$ is employed just for comparison with low-disk mass models, and the comparison should be done in the same simulation setting.

As described in Sec. II A, numerical simulations are carried out typically with the grid spacing of $\Delta x=$ $0.016 M_{\mathrm{BH}}$ near the black hole. This grid resolution is higher than the resolution in previous general relativistic magnetohydrodynamics simulations $[15,16,18,19]$, and this setting enables us to perform a well-resolved simulation for the inner part of the disk (i.e., the thermal and geometrical structure of the disk can be well resolved). For model K8, we perform simulations varying the grid resolution as $\Delta x=0.0133 M_{\mathrm{BH}}$ and $0.020 M_{\mathrm{BH}}$ to confirm the only weak dependence of the numerical results on the grid resolution. 


\section{B. Viscous hydrodynamics of disks for $M_{\text {disk }}=0.1 M_{\odot}$}

This subsection focuses on presenting the results for the models with $M_{\text {disk }}=0.1 M_{\odot}$, except for model D8. Because the disk is more compact for model D8 than for others, the fraction of the disk matter that falls into the black hole is larger and the ejecta mass is smaller than those for the other less-compact disk models. Besides this difference, the numerical results depend only weakly on the initial disk compactness. Thus, we only briefly summarize the results for model D8 in Appendix B, comparing them with those for model $\mathrm{K} 8$, and in this subsection, we only show the results for other models.

Figures 4 and 5 display the evolution of the profiles for the rest-mass density, temperature, specific entropy per baryon, and electron fraction for models $\mathrm{K} 8$ and $\mathrm{K} 8 \mathrm{~h}$. Figure 6(a) plots the evolution of the black-hole mass and dimensionless spin for models $\mathrm{K} 8$ with three different grid resolutions, K8h, and Y8, and Fig. 6(b) shows the total rest mass swallowed by the black hole for all the models with the initial disk mass $M_{\text {disk }}=0.1 M_{\odot}$. The mass and dimensionless spin of the black hole during the evolution are approximately determined in the same method as in Ref. [44]: We calculate the area, $A_{\mathrm{AH}}$, and circumferential radii around the equatorial and meridian planes, $c_{e}$ and $c_{p}$, for the apparent horizon of the black hole, and then estimate the mass and dimensionless spin, assuming that $A_{\mathrm{AH}}, c_{e}$, and $c_{p}$ are written as functions of the mass, $M_{\mathrm{BH}}$, and dimensionless spin, $\chi$, of Kerr black holes as in the vacuum black-hole case.

The first three panels of Fig. 7 show the evolution of the average cylindrical radius $R_{\text {mat }}$, the average value of the specific entropy $\langle s\rangle$, and the average value of $Y_{e},\left\langle Y_{e}\right\rangle$ for the matter located outside the black hole. Here, these average quantities are defined by

$$
\begin{gathered}
R_{\mathrm{mat}}:=\sqrt{\frac{I}{M_{\mathrm{mat}}}}, \\
\langle s\rangle:=\frac{1}{M_{\mathrm{mat}}} \int_{\text {out }} \rho_{*} s d^{3} x, \\
\left\langle Y_{e}\right\rangle:=\frac{1}{\text { mat }} \int_{\text {out }} \rho_{*} Y_{e} d^{3} x,
\end{gathered}
$$

where $\rho_{*}:=\rho \sqrt{-g} u^{t}$, and $I$ and $M_{\text {mat }}$ denote the moment of inertia and rest mass of the matter located outside the black hole, defined by

$$
I:=\int_{\text {out }} \rho_{*}\left(x^{2}+y^{2}\right) d^{3} x
$$

$$
M_{\mathrm{mat}}:=\int_{\mathrm{out}} \rho_{*} d^{3} x
$$

$\int_{\text {out }}$ implies that the volume integral is performed for the matter outside the black hole. The last three panels of Fig. 7 show the total neutrino luminosity, $L_{\nu}$; an efficiency of the neutrino emission defined by the total neutrino luminosity, $L_{\nu}$, divided by the rest-mass energy accretion rate of the matter into the black hole, $c^{2} d M_{\text {fall }} / d t$; and the total ejecta mass $M_{\text {eje }}$ as a function of time. Note that the neutrino luminosity is defined by the total neutrino emission rate minus the neutrino absorption rate, both of which are calculated by the volume integral.

As Figs. 4-7 show, the disk evolves approximately on the viscous timescale defined by Eq. (2.17). We note that the typical value of $c_{s}$ is $0.05 c$ at $R \sim 100 \mathrm{~km}$, and $c_{s}$ is a decreasing function of $R$. In the early stage of the evolution of the disk with the timescale less than $200 \mathrm{~ms}$, a substantial fraction of the inner part of the disk with small values of $R$ falls into the black hole. Irrespective of the models and grid resolutions, $\sim 60 \%-70 \%$ of the initial disk mass, $M_{\text {disk }}$, falls into the black hole during this early stage, and as a result, the black-hole mass increases by $\sim 0.6-0.7 M_{\text {disk }}$, as found in Fig. 6(a). Subsequently, the infall rate significantly decreases [see Fig. 6(b)], and for $t \gtrsim 1 \mathrm{~s}$ the accretion rate onto the black hole becomes smaller than the mass ejection rate (with the mass outflow rate measured for a sphere of $r=2000-4000 \mathrm{~km}$ ), which is typically $\dot{M}_{\text {eje }} \sim 10^{-2} M_{\odot} / \mathrm{s}$ at the peak. Figure 6(a) also shows that for $t \lesssim 200 \mathrm{~ms}$, the dimensionless spin slightly increases due to the matter accretion onto the black hole. We note that the subsequent gradual decrease of $\chi$ is due to the insufficient grid resolution (cf. Sec. III F).

As Figs. 4 and 7(a) show, the outer part of the disk expands outwards in a process lasting more than hundreds of milliseconds by the viscous effect. Since the local viscous timescale for the outer part of the disk with a large value of $R$ is longer than the inner part, the expansion timescale there could be much longer than the timescale of the matter infall to the black hole. However, because the viscous heating/angular momentum transport in the inner part of the disk also contributes to the disk expansion, the outer part of the disk expands on a timescale much shorter than the local viscous timescale of Eq. (2.17). For $\alpha_{\nu}=0.05$, an outer edge of the disk in the equatorial plane with its rest-mass density $\sim 10^{6} \mathrm{~g} / \mathrm{cm}^{3}$ reaches $\sim 10^{3} \mathrm{~km}$ at $t \sim 0.7 \mathrm{~s}$. Simulations for models $\mathrm{K} 8 \mathrm{~h}$ and $\mathrm{K} 8 \mathrm{~s}$ show that this timescale is approximately proportional to $\alpha_{\nu}^{-1}$, as predicted from Eq. (2.17) (e.g., compare Figs. 4 and 5, and see Fig. 7).

For $\alpha_{\nu}=0.05$, the mass ejection sets in (or, strictly speaking, the ejecta component appears) at $\sim 0.5 \mathrm{~s}$ after the onset of the viscous evolution. Before this time, the 

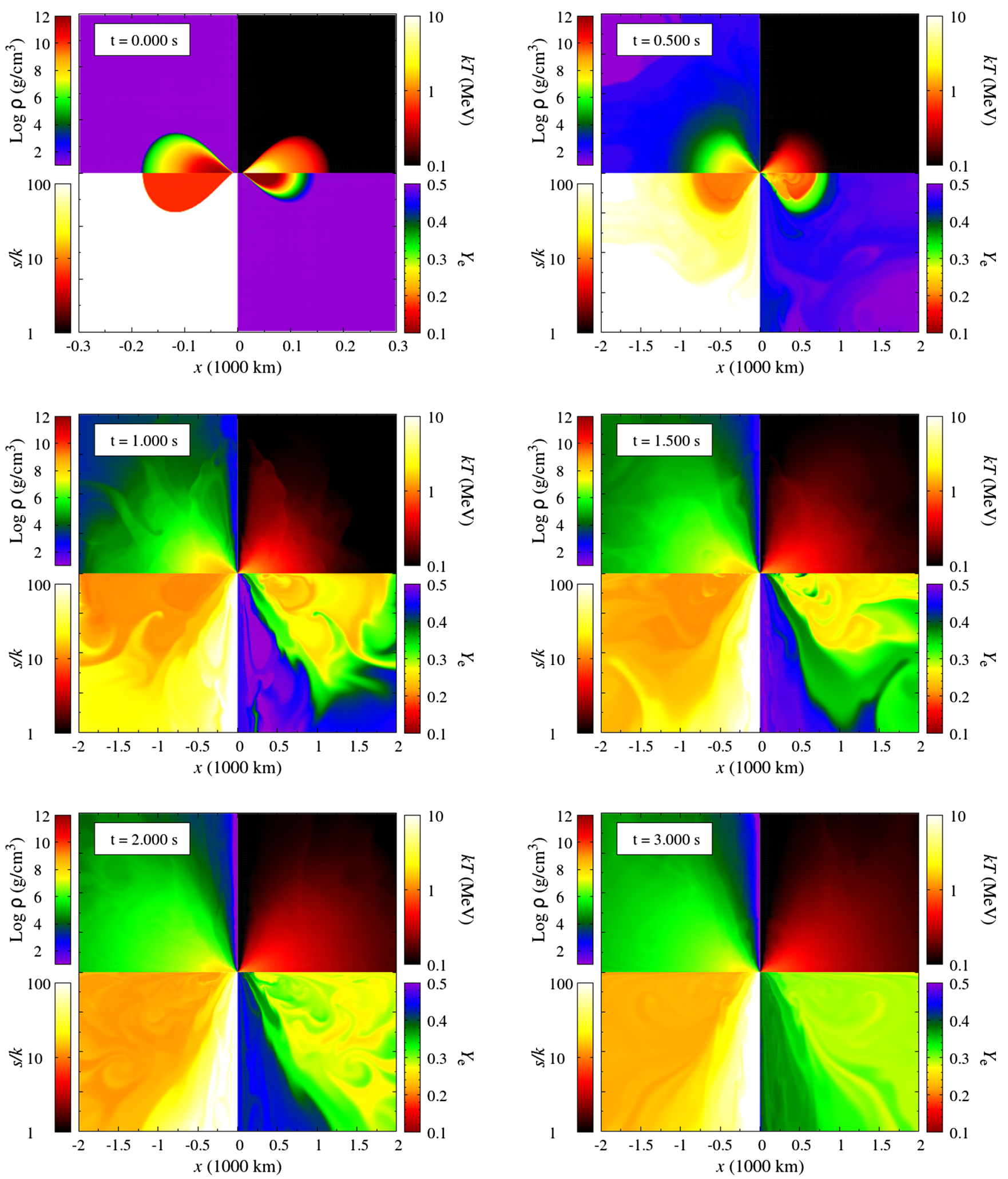

FIG. 4. Snapshots for the rest-mass density in units of $\mathrm{g} / \mathrm{cm}^{3}$, temperature $(k T)$ in units of MeV, specific entropy per baryon in units of $k$, and electron fraction $Y_{e}$ for selected time slices for model K8. Only for the first panel ( $\left.t=0\right)$ is the plotted region $300 \mathrm{~km} \times 300 \mathrm{~km}$; for others, it is $2000 \mathrm{~km} \times 2000 \mathrm{~km}$. We note that the rest-mass density, the value of $Y_{e}$, and the temperature of the atmosphere artificially added are $\approx 10 \mathrm{~g} / \mathrm{cm}^{3}, 0.5$, and $\approx 0.036 \mathrm{MeV} / k$, respectively (cf. the first panel). 

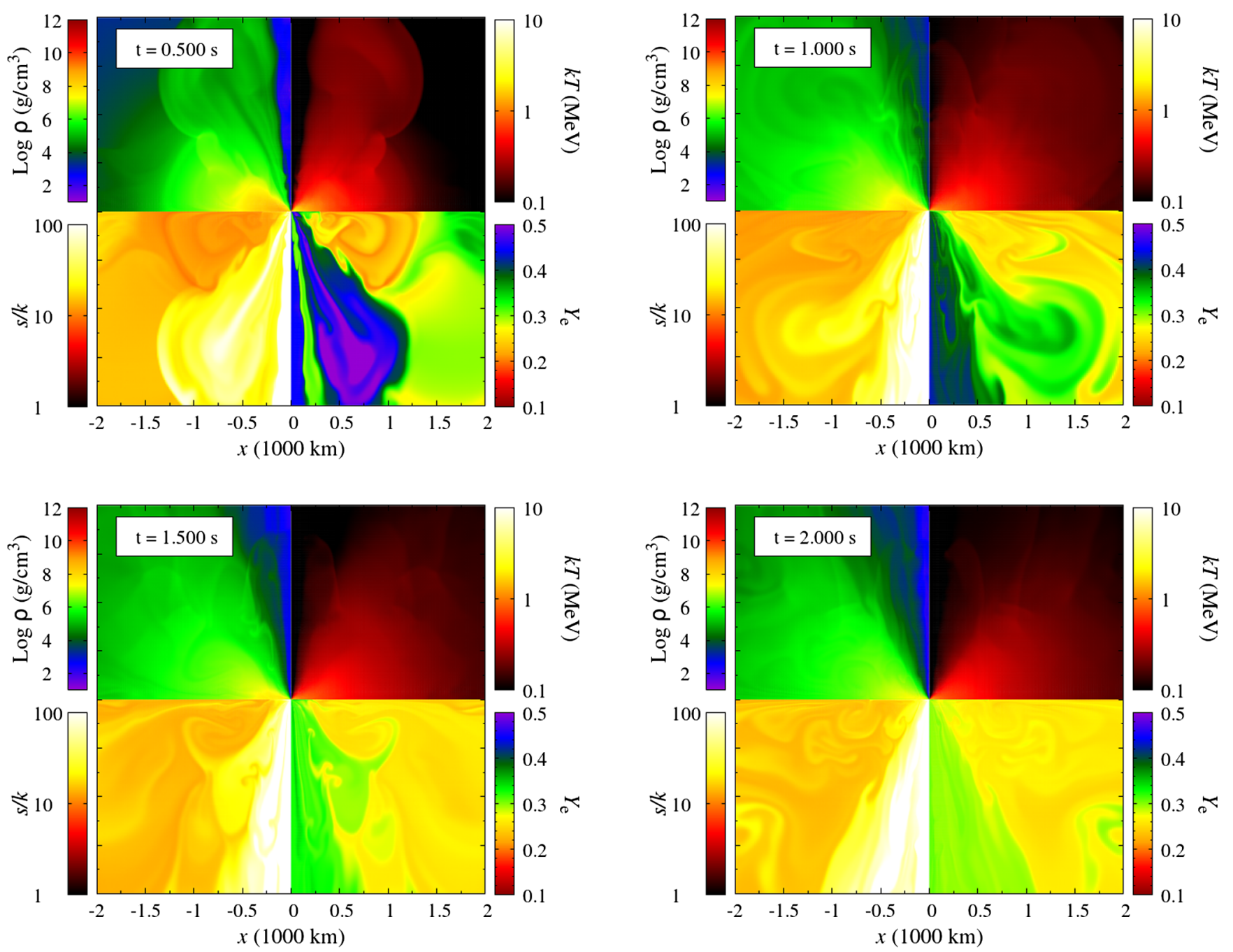

FIG. 5. The same as Fig. 4, but for model K8h.

mass ejection is not activated, as the second panel of Fig. 4 indicates (in this panel, no region with $\rho \gtrsim$ $10^{3} \mathrm{~g} / \mathrm{cm}^{3}$ is found for $r \gtrsim 10^{3} \mathrm{~km}$ ). A signal of the mass ejection is identified by the evolution of $R_{\text {mat }}$ in Fig. 7(a), which shows a steep increase of this quantity from $\sim 300 \mathrm{~km}$ to higher values irrespective of the
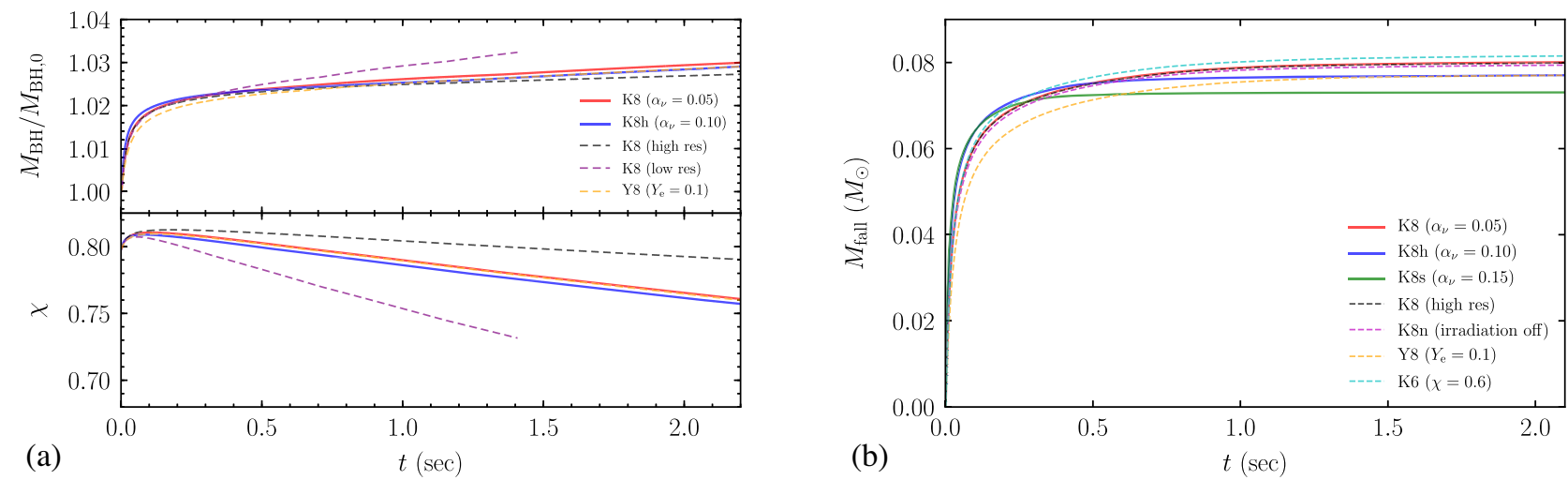

FIG. 6. Left: Evolution of the black-hole mass and dimensionless spin for models K8, K8h, and Y8. For model K8, the results with three different grid resolutions are shown. The gradual decrease of $\chi$ is due to the insufficient grid resolution. Right: Total amount of the rest mass swallowed into the black hole for models $\mathrm{K} 8$ (with two grid resolutions), K8h, K8s, K8n, Y8, and K6. 

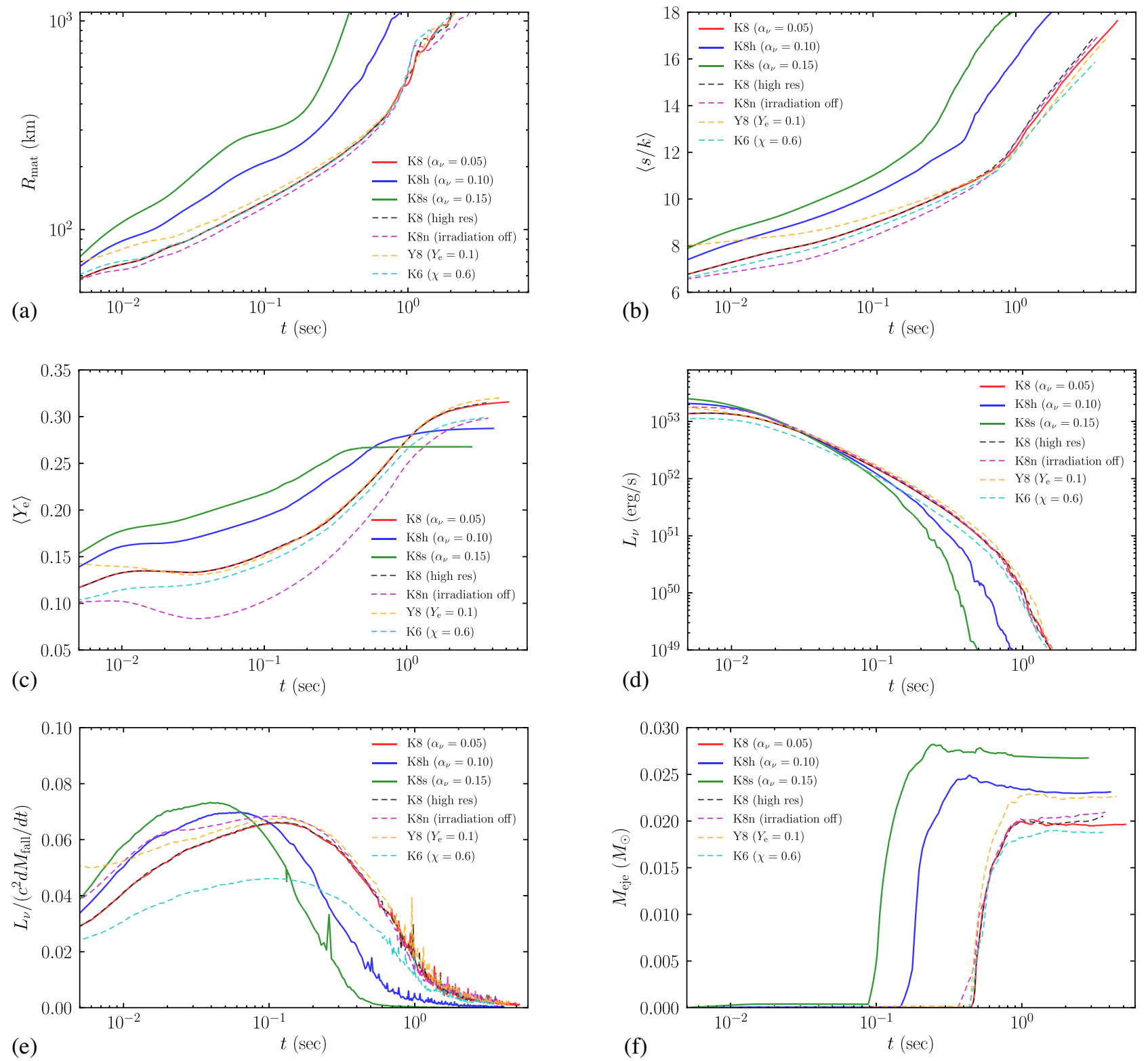

FIG. 7. Several quantities for models K8, K8h, K8n, Y8, and K6. Panels (a)-(c) give the average cylindrical radius (top left), the average specific entropy (top right), and the average value of $Y_{e}$ (middle left) of the matter located outside the black hole as functions of time. Panel (d) shows the time evolution of the total neutrino luminosity, $L_{\nu}$. Panels (e) and (f) show an efficiency of the neutrino emission defined by the total neutrino luminosity, $L_{\nu}$, divided by the rest-mass energy accretion rate of the matter into the black hole, $c^{2} d M_{\text {fall }} / d t$ (bottom left) and the total mass of the ejecta component $M_{\text {eje }}$ (bottom right), both as functions of time. For model K8, the results with a higher-resolution run are also plotted and show their weak dependence on the grid resolution.

models. The primary driving force of the mass ejection is the viscous heating in the inner region of the disk under the negligible neutrino cooling. For $t \gtrsim 0.5 \mathrm{~s}$, the disk has already expanded by the gradual viscous heating/angular momentum transport enough to decrease its temperature $(k T)$ below $\sim 2 \mathrm{MeV}$ (see Fig. 4). As a result, the neutrino cooling does not play an important role (the neutrino cooling timescale becomes longer than the viscous timescale; see Appendix A), and thus, the viscous heating is fully used for the disk heating and mass ejection $[10,14]$.
Indeed, the mass ejection efficiency is enhanced when the neutrino luminosity decreases below a certain threshold, as observed in Figs. 7(d) and 7(f). By contrast, in the early disk evolution-in particular, for $t \lesssim 100 \mathrm{~ms}$, during which the total neutrino luminosity is $\gtrsim 10^{52} \mathrm{erg} / \mathrm{s}-$ the viscous heating is mostly consumed by the neutrino emission, and thus the viscous effect cannot have the power to eject matter. Indeed, the viscous heating rate, $\sim \nu M_{\text {disk }} \Omega^{2}$, is of the same order of $L_{\nu}$ for the typical values of $\nu, M_{\text {disk }}$, and $\Omega$ of the disk. 
The viscous heating is always most efficient in the innermost region of the disk (i.e., the region closest to the black hole). The enhancement of the specific entropy then triggers convective motion from the innermost region toward outer regions, after the neutrino cooling becomes inefficient. We evaluated the Solberg-Holland frequency for the convective instability $[45,46]$, and we found that the innermost region of the disk at $r \sim 100-150 \mathrm{~km}$ near the equatorial plane is indeed unstable to the convective motion. The frequency of the convective instability in the innermost region is $\sim(10 \mathrm{~ms})^{-1}$, and hence the timescale for the convective motion is much shorter than the viscous timescale.

However, the convective activity is suppressed by the centrifugal force, which tends to stabilize the convective motion. This stabilization effect is strong in the vicinity of the main body of the disk with the highest density. As a result, the matter of the high specific entropy produced in the innermost region goes along a high-latitude region of the disk. Nevertheless, the high-entropy convective blob eventually brings the thermal energy into the outer part of the disk with a large cylindrical radius and with a high latitude. By the increase of the thermal energy there, the matter in the outer part of the disk obtains enough energy to escape from the system as ejecta. Therefore, the onset time of the mass ejection is determined approximately by the viscous heating timescale for the innermost region of the disk at the moment that the neutrino emission timescale becomes as long as the viscous heating timescale, and the major process of energy transport is convection (cf. Ref. [47]). By convective activity, the matter is ejected in any direction except along the rotation axis (see Sec. III D).

We note that the viscous heating/angular momentum transport in the high-density region of the disk also contributes to the expansion of the entire region of the disk. Thus, not only the convective motion but also the continuous viscous effect and resulting disk expansion play a role for the mass ejection. This is in particular the case for the high viscous coefficients, as indicated in Sec. IIID. In addition, the thermal energy stored in the innermost region by the viscous heating drives an intermittent wind. This contributes to nonsteady mass ejection -in particular, for the late-time evolution of the disk - in which the neutrino cooling plays a negligible role and viscous heating can be fully available for driving the wind.

To examine the significance of the neutrino heating (irradiation) effect, we also performed a simulation without neutrino heating (model K8n). Our result shows that neutrino heating does not play a substantial role for the mass ejection in the present fiducial model; the mass ejection rate and ejecta velocity are not substantially influenced by the neutrino heating. The reason for this is that the neutrino luminosity is not very high during the mass ejection stage in our model [see Fig. 7(d)]: Only in the early stage of the
TABLE II. Rest mass, average value of velocity, and average value of $Y_{e}$ for ejecta. All the quantities are extracted at $t=3.5 \mathrm{~s}$ after the onset of the simulations except for models K8s and $\mathrm{C} 8$, for which the time for the extraction is $t=2.5 \mathrm{~s}$ and $6.0 \mathrm{~s}$, respectively. The last column shows the lanthanide mass fraction determined by the nucleosynthesis calculation for selected models.

\begin{tabular}{lcccc}
\hline \hline Model & $M_{\mathrm{ej}}\left(M_{\odot}\right)$ & $V_{\text {ave }} / c$ & $Y_{e, \text { ave }}$ & $X_{\text {lan }}$ \\
\hline K8 & 0.020 & 0.06 & 0.31 & $2.8 \times 10^{-4}$ \\
K8 (high resolution) & 0.020 & 0.06 & 0.31 & \\
K8h & 0.023 & 0.08 & 0.28 & $1.6 \times 10^{-3}$ \\
K8s & 0.027 & 0.08 & 0.26 & $8.4 \times 10^{-3}$ \\
K8n & 0.021 & 0.07 & 0.30 & \\
D8 & 0.015 & 0.06 & 0.31 & \\
Y8 & 0.023 & 0.06 & 0.32 & \\
K6 & 0.019 & 0.06 & 0.30 & \\
C8 & 0.092 & 0.06 & 0.32 & $1.2 \times 10^{-6}$ \\
E8 & 0.006 & 0.07 & 0.31 & $1.5 \times 10^{-3}$ \\
\hline \hline
\end{tabular}

disk evolution with $t \lesssim 20 \mathrm{~ms}$ does the total luminosity exceed $10^{53} \mathrm{erg} / \mathrm{s}$, while for $t>100 \mathrm{~ms}$, for which the mass ejection becomes active, the luminosity drops below $10^{52} \mathrm{erg} / \mathrm{s}$ exponentially, resulting in a small contribution to the mass ejection by the neutrino heating. ${ }^{1}$ This result is in broad agreement with that of Ref. [14].

After the onset of the convective motion at $t \sim 0.5 \mathrm{~s}$ (for $\alpha_{\nu}=0.05$ with/without neutrino irradiation), the viscous heating and resulting convection continue to play a leading role for the mass ejection, although the mass ejection rate gradually decreases with decreasing disk density. The total ejecta mass is $15 \%-30 \%$ of the initial disk mass (see Fig. 7 and Table II). For a fixed viscous coefficient with $\alpha_{\nu}=0.05$, the ejecta mass is $\approx 15 \%-25 \%$ irrespective of the initial condition (i.e., initial $Y_{e}$ distribution, density and velocity profiles, compactness of the disk, and blackhole spin). The fraction of the ejecta mass agrees broadly

\footnotetext{
${ }^{1}$ We note that the maximum energy obtained from neutrinos per nucleon (neutron or proton) via neutrino absorption is estimated approximately as $\Delta E_{\nu} \sigma_{\nu} /\left(4 \pi r^{2}\right)$, where $\sigma_{\nu}$ is the cross section of nucleons with neutrinos and $\Delta E_{\nu}$ is the total energy emitted by each neutrino species (here electron neutrinos or antineutrinos). In model K8, $\Delta E_{\nu} \sim 5 \times 10^{52} \mathrm{erg} / \mathrm{s} \times 0.02 \mathrm{~s} \sim 10^{51} \mathrm{erg}, \sigma_{\nu} \sim$ $8 \times 10^{-42} \mathrm{~cm}^{2}$ for neutrinos with energy $\sim 10 \mathrm{MeV}$, and $4 \pi r^{2} \gtrsim$ $10^{13} \mathrm{~cm}^{2}$ because $r \gtrsim 2 G M_{\mathrm{BH}} / c^{2} \sim 9 \mathrm{~km}$. If we do not consider neutrino cooling, the maximum energy obtained from neutrinos would be $\sim 800\left(2 G M_{\mathrm{BH}} / c^{2} r\right)^{2} \mathrm{MeV}$ per nucleon. In reality, the energy gain of each nucleon is much smaller than this, because the cooling by the neutrino emission could be of the same order of magnitude as the heating. However, even if we ignore the neutrino cooling, the obtained energy gain is at most as large as the gravitational potential energy of nucleons, $\sim G M_{\mathrm{BH}} m_{n} / r$, where $m_{n} c^{2}$ is the rest-mass energy of nucleons $\sim 940 \mathrm{MeV}$. In particular, for $r \geq 10 G M_{\mathrm{BH}} / c^{2}$, the estimated energy gain is more than 1 order of magnitude smaller than the gravitational potential energy.
} 
with the results of the earlier viscous hydrodynamics work $[10,11,14]$.

The entropy and electron fraction in the disk increase with its viscous and convective expansion after the initial quick matter accretion onto the black hole for $t \lesssim 100 \mathrm{~ms}$ ceases [see Figs. 7(b) and (c)]. The typical average value of the specific entropy is $10-12 k$ when the mass ejection is activated. After the initial infall stage of the disk matter onto the black hole, the average value of $Y_{e}$ monotonically increases with decreasing disk density, and it is higher than $\sim 0.2$ at the onset of the mass ejection, irrespective of the models for $\alpha_{\nu}=0.05$. At late times, the average value of $Y_{e}$ settles to constants of $\sim 0.3$. The mechanism for this $Y_{e}$ evolution is summarized as follows: During the disk expansion ongoing until the weak interaction freezes out (i.e., the temperature is $k T \gtrsim 2 \mathrm{MeV}$ and the electron degeneracy is not so strong that the weak interaction rates are determined predominantly by the temperature: see Appendix A), the weak interaction processes determine the electron fraction in the disk (the value of $Y_{e}$ is approximately determined by the equality of the rates of electron/positron capture on nucleons; see Appendix A for more details). However, for $k T \lesssim 2 \mathrm{MeV}$, the weak interaction plays a negligible role, because the timescale for the weak interaction processes, $\tau_{\beta}$, becomes longer than the viscous timescale, $\tau_{\text {vis }}[10]$ (here $\tau_{\beta}$ is approximately equal to the neutrino emission timescale). Thus, the settled value of $Y_{e}$ is determined approximately by the condition of $\tau_{\beta}=\tau_{\text {vis }}$. The onset time of this freeze-out of the weak interaction agrees approximately with the time that the mass ejection is activated. In our numerical result, the relaxed average values of $Y_{e}$ are not very small, i.e., $\gtrsim 0.25$, irrespective of the models for $\alpha_{\nu}=0.05$. This has an important implication for the nucleosynthesis of lanthanide elements, as we discuss in Sec. III E.

Figure 7(e) shows that the total neutrino luminosity for $\chi=0.8$ is $\sim 6 \%-7 \%$ of the rest-mass energy accretion rate onto the black hole at the maximum. This efficiency agrees with that in Ref. [14]. The maximum efficiency is achieved in an early stage of the disk evolution in which the neutrino luminosity is high and the weak interaction timescale is still shorter than the viscous evolution timescale. This efficiency, as well as the neutrino luminosity, is smaller for $\chi=0.6$ than for $\chi=0.8$. The reason for this is that the radius of the innermost stable circular orbit is larger for the smaller value of $\chi$, and thus the depth of the gravitational potential in the vicinity of the black hole is shallower; i.e., the gravitational potential energy available for the dissipation is smaller. For higher viscous coefficients, the efficiency is higher, but the enhancement is not very remarkable. Thus, the maximum efficiency is basically determined by the spin of the black hole. In the late stage of the disk evolution in which the weak interaction freezes out, the efficiency approaches zero, because the emissivity of neutrinos exponentially drops. As already mentioned, in this late stage, the mass ejection is enhanced.

Figure 5 displays the evolution of the profiles for model K8h-i.e., for a larger-viscosity model than model K8. For $\alpha_{\nu}=0.10$ and 0.15 (the $\mathrm{K} 8 \mathrm{~h}$ and $\mathrm{K} 8 \mathrm{~s}$ models), the mass ejection sets in earlier than for model K8. This is clearly found by comparing the profiles at $t=0.5 \mathrm{~s}$ in Figs. 4 and 5. The reason for the earlier onset of the mass ejection is simply that the viscous timescale becomes shorter, the density of the disk decreases more quickly, and hence the freeze-out of the weak interaction occurs earlier. Another remarkable difference among the three models $\mathrm{K} 8, \mathrm{~K} 8 \mathrm{~h}$, and $\mathrm{K} 8 \mathrm{~s}$ is that the mass ejection from the inner edge of the disk as a disk wind is more powerful for the higherviscosity models. This occurs because the thermal pressure by the viscous heating and also the viscous angular momentum transport for both $\alpha_{\nu}=0.10$ and 0.15 are more enhanced than for $\alpha_{\nu}=0.05-$ in particular, in the innermost region of the disk.

Associated with the stronger viscous heating, the specific entropy and the average value of $Y_{e}$ in the disk increase more rapidly [see Figs. 7(b) and 7(c)]. The reason for this is that the temperature of the disk is higher and the electron degeneracy is weakened at a given time for the higherviscosity models. As we mentioned above, however, the disk expansion occurs more quickly for the larger viscous coefficients. As a result, the weak interaction freezes out earlier, and also the electron fraction settles to smaller values for the larger viscous coefficients [compare the panels in Figs. 4 and 5 at $t=2 \mathrm{~s}$ and also see Fig 7(c)]. This modifies the $Y_{e}$ distribution of the ejecta, as discussed in Sec. III D.

The larger viscous effect slightly suppresses the matter infall into the black hole [see Fig. 6(b)]. Associated with this, the total ejecta masses for $\alpha_{\nu}=0.10$ and 0.15 become larger than that for $\alpha_{\nu}=0.05$ by $0.004 M_{\odot}$ and $0.007 M_{\odot}$, respectively [see Figs. 7(e) and 7(f), as well as Table II]. All these results show that the magnitude of the viscous coefficient significantly influences the disk evolution and the quantitative properties of the ejecta.

Figure 7 clearly shows that the effect of the black-hole spin is not substantial on the evolution of the system for the astrophysically plausible values of $\chi$ as the merger remnants (compare the results of models K8 and K6). We can find that the average value of $Y_{e}$ and the neutrino luminosity for model K6 are slightly smaller than those for model $\mathrm{K} 8$, because the innermost edge of the disk for model K6 is located slightly farther outside the black hole than for model K8, and as a result, the viscous heating efficiency and resulting ejecta mass are slightly smaller. However, this spin effect is minor for modifying the evolution of the system: The effect in the change of the viscous coefficient by a factor of 2 has a much stronger impact. 


\section{Viscous hydrodynamics of disks: Comparison among different disk mass models}

The difference in the disk mass (compare models $\mathrm{K} 8$, $\mathrm{C} 8$, and E8) results in the modification of the timescale to reach the freeze-out of the weak interaction and onset time of the mass ejection. Figure 8 displays the snapshots of the profiles for the rest-mass density, temperature, specific entropy per baryon, and electron fraction for model C8 at selected time slices. Figure 9 also compares the average cylindrical radius, average specific entropy, average value of $Y_{e}$ for the matter outside the black hole, total neutrino luminosity, an efficiency of the neutrino emission, $L_{\nu} /\left(d M_{\text {fall }} / d t\right)$, and ejecta mass, as well as the total mass swallowed by the black hole among models $\mathrm{K} 8, \mathrm{C} 8$, and E8. These figures show that for a larger disk mass, the density and temperature are always higher at a given time, and the freeze-out time of the weak interaction comes later. As a result, for the more massive models, the thermal energy generated by the viscous heating is released by the neutrino emission for a longer term, resulting in more luminous and long-lasting neutrino emission. Then, the disk expansion timescale becomes longer [see Fig. 9(a)], the onset time of the convection becomes later, and the mass ejection is delayed [14]. These facts are well reflected in the maximum efficiency of the neutrino emission and the duration of the high-luminosity neutrino emission [see Figs. 9(c) and (e)]. Due to the delay of the freeze-out of the weak interaction, the average value of $Y_{e}$ in the disk at the freeze-out of the weak interaction becomes higher for more massive disk models [see Fig. 9(c)]. Figure 8 also illustrates that besides the quantitative difference mentioned above, the evolution process of the disk is qualitatively similar among different disk-mass models; e.g., the mass ejection is driven primarily by the convective activity caused by the viscous heating in the region of the disk close to the black hole for all the models.

For the remnant of neutron-star mergers, disks with masses of $\gtrsim 0.5 M_{\odot}$ are not very likely. However, such a heavy disk surrounding a spinning black hole could be formed as a result of rotating massive stellar core collapses, in particular as a central engine of long gamma-ray bursts [48]. Such heavy disks are also likely to be evolved effectively by viscous hydrodynamics processes. Our present numerical results demonstrate that for large disk masses, the electron fraction at which the weak interaction freezes out could be large, with $Y_{e} \gtrsim 0.3$ (unless the viscous coefficient is extremely large). Thus, if the viscous process is the dominant mechanism of mass ejection, the matter ejected from the disk may not be very neutron rich for the remnant of stellar core collapses (see also the discussion in the next sections).

\section{Properties of ejecta}

Figure 10 shows the average value of $Y_{e}$ and the average velocity of the ejecta as functions of time for all the models (except for model D8) employed in this paper. Here, we plot the ejecta velocity determined only for the ejecta component that escapes from the sphere of $r=2000 \mathrm{~km}$. We note that even if the extraction radius is changed from $r=2000 \mathrm{~km}$ to $3000 \mathrm{~km}$ or $4000 \mathrm{~km}$, the asymptotic values of $Y_{e}$ and the velocity are varied only within 3\%. Table II lists the rest mass, average velocity, and average value of $Y_{e}$ of the ejecta for all the models considered in this paper. We also show the mass histogram (mass fraction) of the ejecta as a function of $Y_{e}$ for models K8, K8h, K8s, K8n, Y8, K6, C8, and E8 in Fig. 11. Here, the value of $Y_{e}$ is determined at the time when the temperature of the corresponding ejecta component decreases to $5 \times 10^{9} \mathrm{~K}$ (referred to as $5 \mathrm{GK}$ : note that the temperature of the ejecta component in general decreases down to $\sim 3 \mathrm{GK}$ monotonically during the ejection process).

The average values of $Y_{e}$ for the ejecta with $\alpha_{\nu}=0.05$ are $\sim 0.3$ and depend very weakly on the initial condition for the disk profiles of density, angular velocity, and $Y_{e}$. On the other hand, it decreases significantly with the increase of the viscous coefficient. As Fig. 11(a) shows, the distribution shifts to the lower side of $Y_{e}$ with the increase of $\alpha_{\nu}$. This result can be expected from Fig. 7(c), which shows that for the matter outside the black hole, the asymptotic average values of $Y_{e}$ are $\sim 0.32,0.29$, and 0.27 for $\alpha_{\nu}=0.05,0.10$, and 0.15 , respectively. These values agree approximately with the average value of $Y_{e}$ for the ejecta. This agreement indicates that the matter in the outer part of the disk that expands by the viscous effect and the resulting convective heating eventually becomes the ejecta component. The average value of $Y_{e}$ for the ejecta increases with the increase of the disk mass (compare the results for models $\mathrm{C} 8, \mathrm{~K} 8$, and $\mathrm{E} 8$ ). This correlation also agrees with that found in Fig. 9(c).

The asymptotic average velocity of the ejecta, $v_{\text {eje }}$, is $\sim 0.06 c$ for $\alpha_{\nu}=0.05$ and again depends very weakly on the initial condition for the disk profiles of density, angular velocity, $Y_{e}$, and the black-hole spin. For higher viscous coefficients, $v_{\text {eje }}$ is increased, and for $\alpha_{\nu}=0.10$ and 0.15 , it is $\approx 0.08 c$. This reflects an efficient acceleration of the matter in the outer part of the disk for the higher viscous coefficients.

Although the results for the mass and velocity of the ejecta agree broadly with those of the earlier numerical studies by other groups $[10,11,14]$, our result for the mass distribution of $Y_{e}$ shows a noticeable difference from the previous results. In the previous results, a substantial fraction of the ejecta has rather small values of $Y_{e}$ between 0.1 and 0.2. By contrast, our results show that such low $Y_{e}$ components are rare, and the peak is located approximately between $Y_{e}=0.25$ and 0.30 . We note that the low $Y_{e}$ component is present in the disk for the early stage in which the disk density is high, $\gtrsim 10^{9} \mathrm{~g} / \mathrm{cm}^{3}$ (see, e.g., Fig. 4 at $t=0.5 \mathrm{~s}$ ), but with decreasing disk density, the value of $Y_{e}$ 

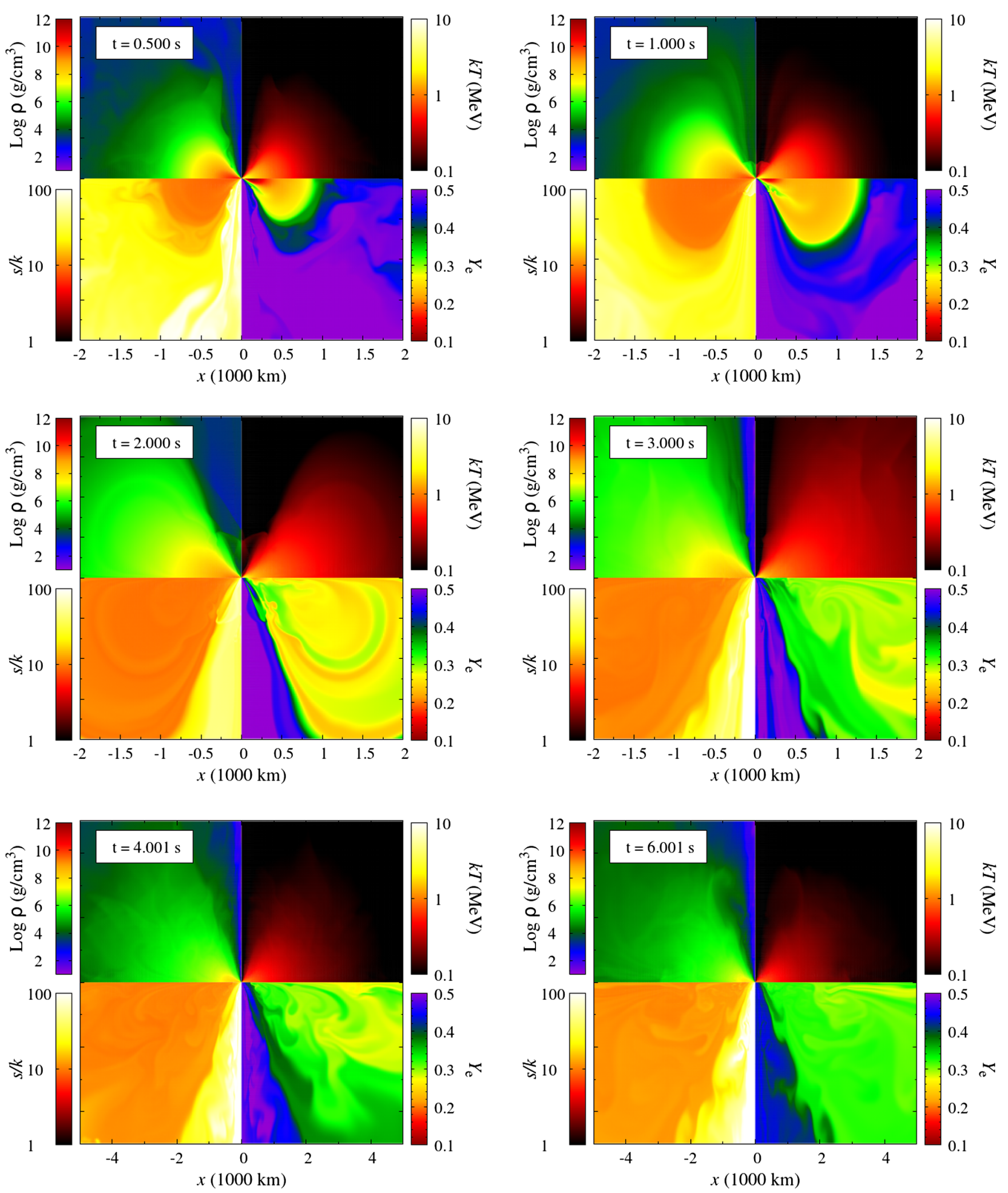

FIG. 8. The same as Fig. 4, but for model C8. For the last two panels at $t=4$ and $6 \mathrm{~s}$, the plots are made for an enlarged region of $5000 \mathrm{~km} \times 5000 \mathrm{~km}$. 


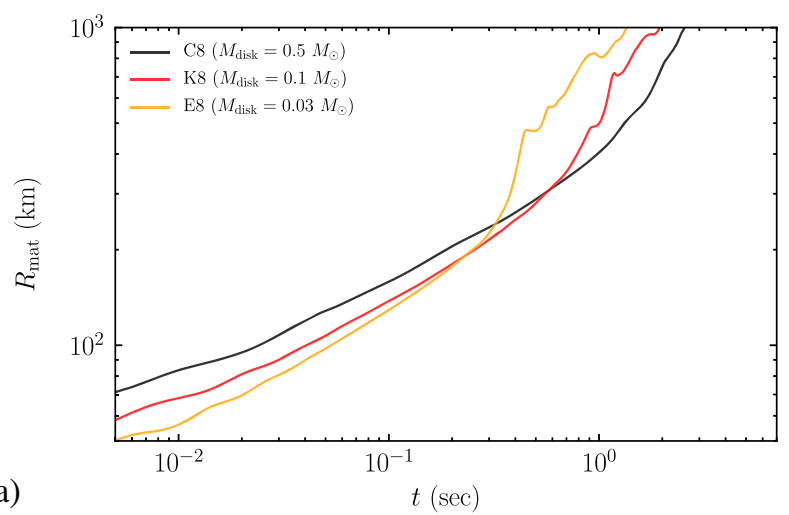

(a)
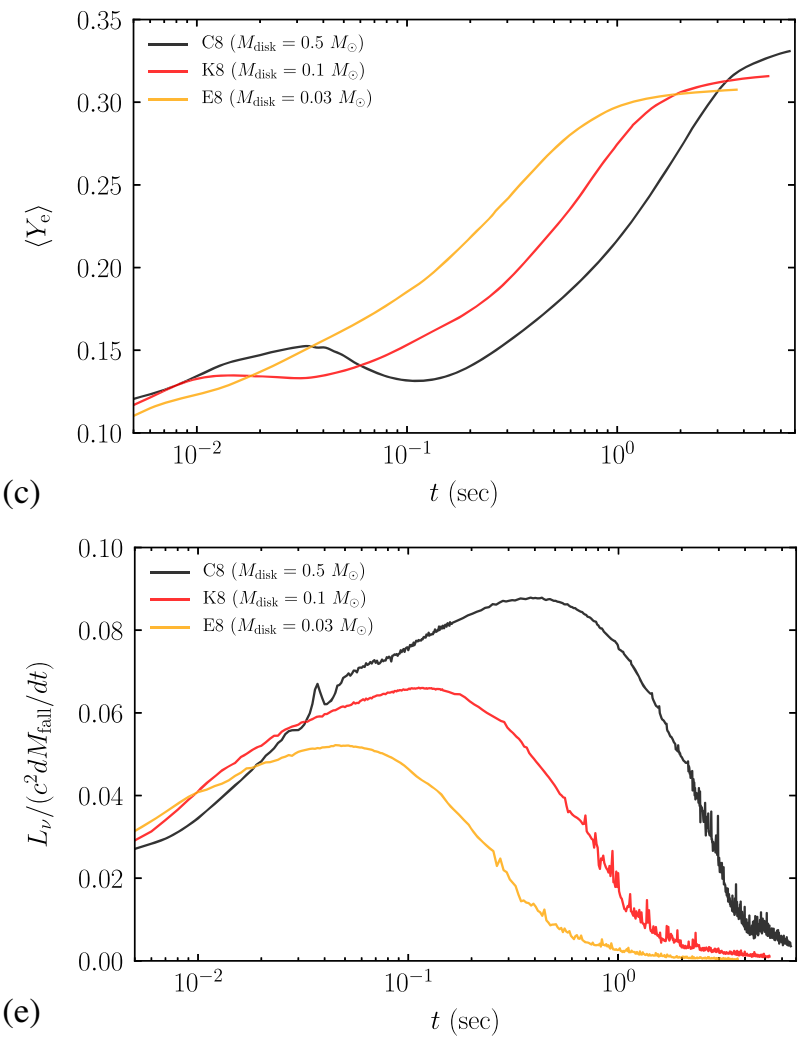
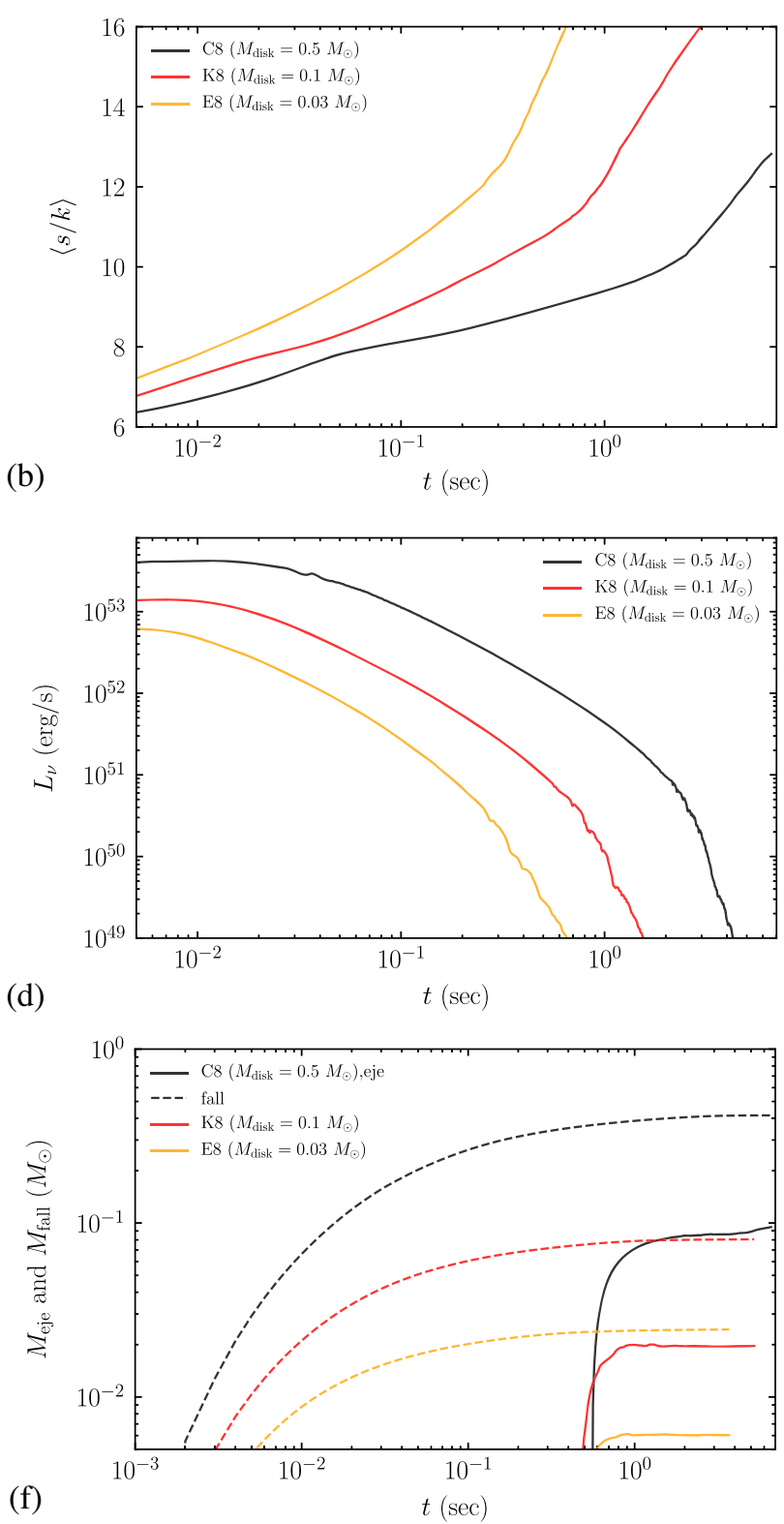

FIG. 9. The same as Fig. 7, but for comparison among different disk mass models C8, K8, and E8. In the bottom-right panel, not only the total ejecta mass (solid curves) but also the total mass that falls into the black hole (dashed curves) is plotted.

is increased, and the ejecta does not have the component with $Y_{e} \leq 0.2$. One possible reason for the difference of our results from the previous ones is that the equation of state and/or treatment of the weak interaction (e.g., electron and positron capture) that we employ may be different from those in the previous studies. Another reason is that in our simulation we do not have any mechanism for the mass ejection which is more efficient than the viscosity-driven ejection. In the following, we describe these points in more detail.

In our simulation, the mass ejection occurs only after the viscous heating and angular momentum transport proceed at least for several hundreds of milliseconds, which causes the matter in the disk to expand to $r \gtrsim 10^{3} \mathrm{~km}$. We do not find any other major components for mass ejection like the neutrino-wind component, which is found in Ref. [14]. As we already mentioned in Sec. III B, in this viscous evolution process, associated with the decrease of the density and with the decrease of the degree of electron degeneracy, the value of $Y_{e}$ in the disk gradually increases from a low value of $Y_{e} \sim 0.1$ to higher values $Y_{e} \gtrsim 0.2$ until the weak interaction freezes out (i.e., the temperature of the disk decreases below $k T \sim 2 \mathrm{MeV}$; e.g., see Fig. 7 and Appendix A). The value of $Y_{e}$ is determined approximately by the condition that electron and positron capture rates are identical. After the freeze-out of the weak interaction, the average of $Y_{e}$ approaches asymptotically $\sim 0.3$ (for $\alpha_{\nu}=0.05$ ). This asymptotic value is slightly larger than 

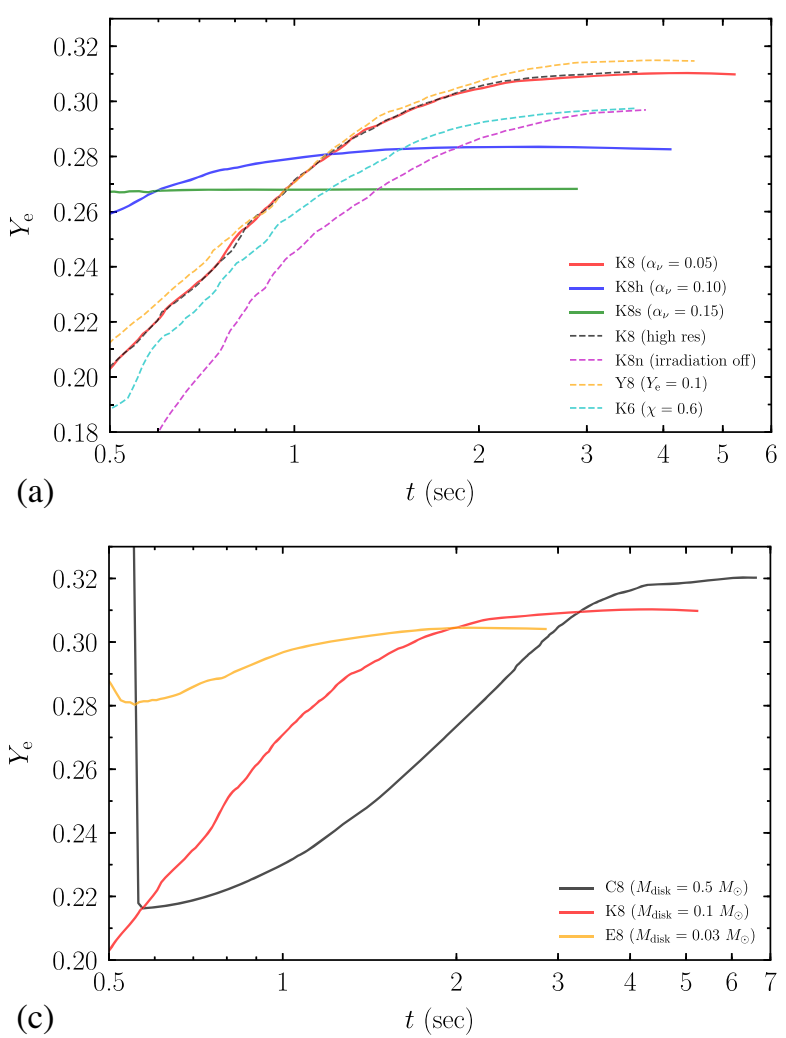
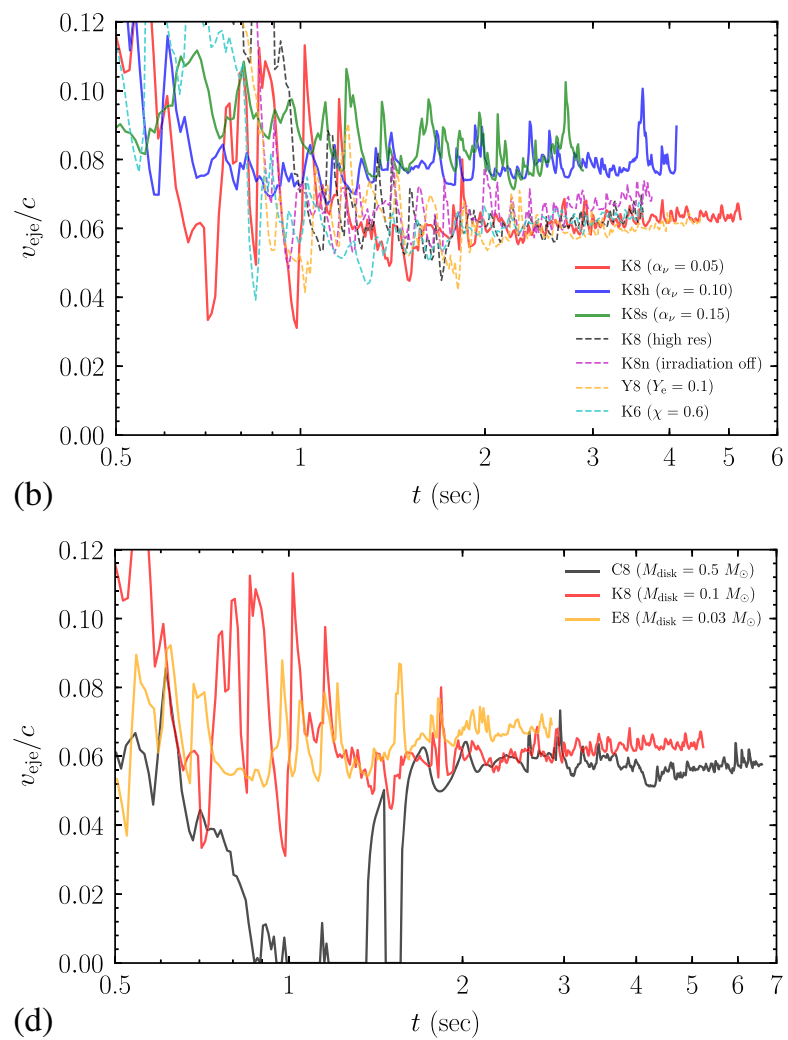

FIG. 10. Average value of $Y_{e}$ (left) and average velocity (right) of the ejecta as functions of time for models with $M_{\text {disk }}=0.1 M_{\odot}$ (top) and for models $\mathrm{C} 8, \mathrm{~K} 8$, and E8 (bottom). Here, the average velocity is determined only for the ejecta component that escapes from $r=2000 \mathrm{~km}$.

that in a previous study [14], and this suggests that a difference in the equation of state, treatment of the weak interaction, and initial thermodynamical condition (e.g., temperature) might result in a difference in the values of $Y_{e}$ among different groups.

For the late stage with lower temperatures of $k T \lesssim 2 \mathrm{MeV}$, the weak interaction does not play a role any longer, because the timescale for the weak interaction process becomes longer than the viscous timescale (see Appendix A). This results in the freeze-out of the value of $Y_{e}$ in the disk. Since the $Y_{e}$ distribution of the ejecta is just the reflection of that in the disk, the value of $Y_{e}$ for the ejecta thus determined is not very small but rather large as $\gtrsim 0.25$ (for $\alpha_{\nu}=0.05$ ). We note that in our simulation, most of the ejecta component experiences the weak interaction process during the viscous evolution of the disk, resulting in a relatively high value of $Y_{e}$. This indicates that the low $-Y_{e}$ ejecta with $Y_{e} \sim 0.1$ found in the previous studies would be driven before experiencing the weak interaction processes sufficiently.

For higher values of $\alpha_{\nu}$, the weak interaction freezes out earlier (much earlier than $1 \mathrm{~s}$ ), because faster expansion occurs for the disk. Since the duration to increase the value of $Y_{e}$ is shorter, $Y_{e}$ becomes smaller for larger values of $\alpha_{\nu}$. This tendency is clearly found by comparing the mass histogram as a function of $Y_{e}$ for models $\mathrm{K} 8, \mathrm{~K} 8 \mathrm{~h}$, and $\mathrm{K} 8 \mathrm{~s}$ [see Fig. 11(a)]. This suggests that in the presence of a mass ejection process more efficient than the viscosity-driven mechanism, like a magnetohydrodynamics mechanism, which would be primarily not the MRI but the magnetic winding and resulting strong Lorentz force with a hypothetically very large poloidal magnetic field aligned with the black-hole spin direction [18], the matter with even lower $Y_{e}$ components may be ejected. However, our present simulations show that in the absence of such an efficient mass ejection process with the ejection timescale much shorter than 1 s (i.e., only with the viscous process), the value of $Y_{e}$ for the ejecta can be quite large, as $Y_{e} \gtrsim 0.2$ even in the absence of strong neutrino irradiation sources, like a remnant massive neutron star [20].

Figure 11(b) compares the mass histogram for models $\mathrm{K} 8$ and Y8. This shows that for model Y8, a small fraction of low $-Y_{e}$ ejecta components with $Y_{e} \leq 0.2$ is present, reflecting its initial condition and the fact that a small fraction of matter is spuriously ejected in the initial transient phase until the disk relaxes to a quasistationary state. However, for the major part of the ejecta, the distribution for $Y_{e}$ is quite similar between these two models. This indicates that the $Y_{e}$ distribution of the ejecta depends only weakly on its initial condition, if we focus only on the viscosity-driven ejecta. We note, however, that 

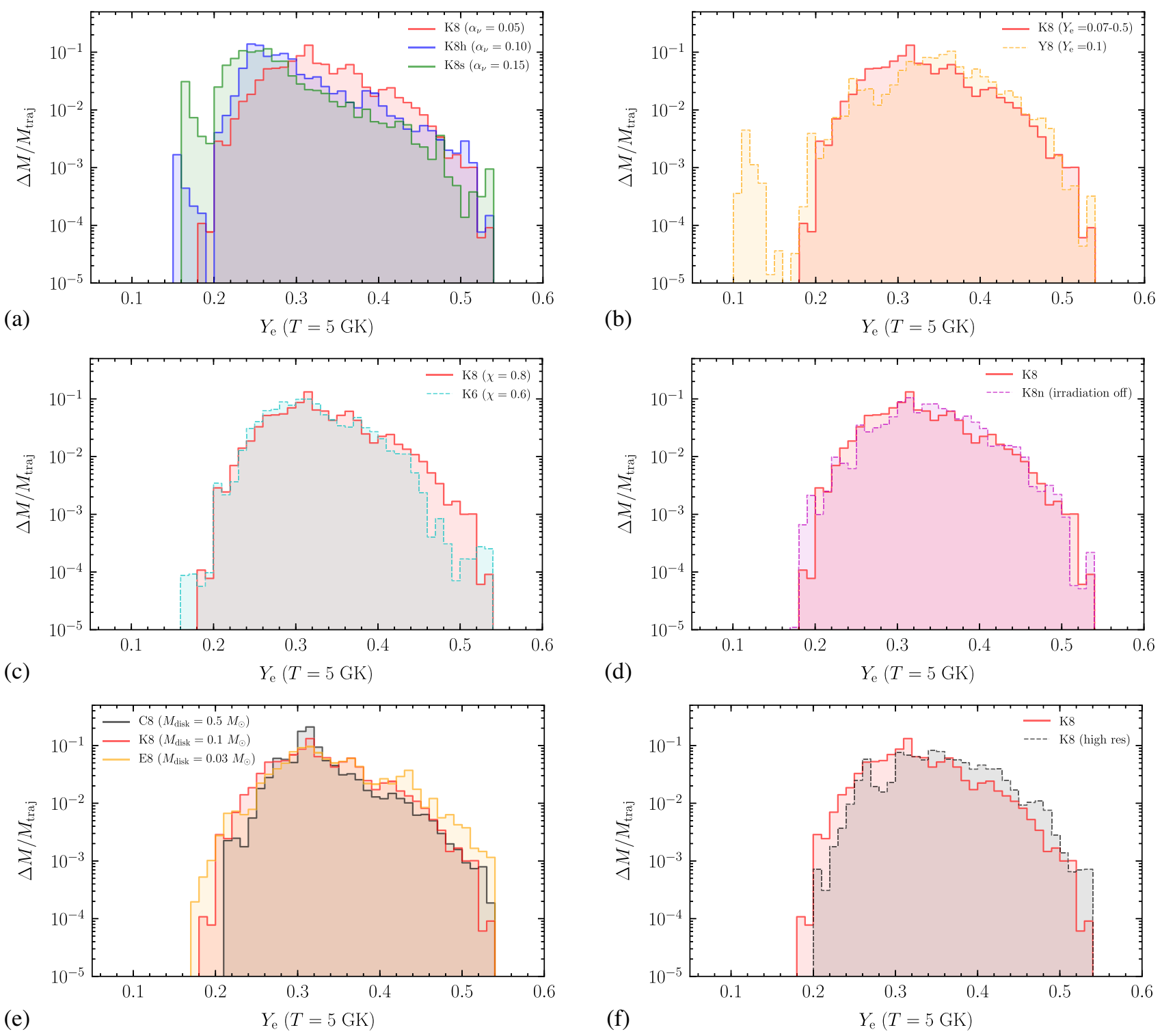

FIG. 11. Mass histogram (mass fraction) of the ejecta as a function of $Y_{e}$ for models (a) K8, K8h, and K8s; (b) K8 and Y8; (c) K8 and $\mathrm{K} 6$; (d) $\mathrm{K} 8$ and $\mathrm{K} 8 \mathrm{n}$; (e) C8, K8, and E8; and (f) $\mathrm{K} 8$ with two grid resolutions. Here, the value of $Y_{e}$ is determined at the time when the temperature of each ejecta component decreases to $5 \times 10^{9} \mathrm{~K}$ (referred to as $5 \mathrm{GK}$ ).

if a very efficient mechanism of mass ejection is present in the very early stage of the disk evolution for $t<100 \mathrm{~ms}$, the resulting mass histogram as a function of $Y_{e}$ may reflect the difference of the initial condition. This point should be explored in the simulation community.

Figure 11(c) compares the mass histograms for models K8 and K6-i.e., for different black-hole spin models. This figure shows that for a smaller black-hole spin (K6), the $Y_{e}$ distribution shifts slightly to the lower- $Y_{e}$ side. Our naive interpretation for this is that for the smaller spin, the disk mass becomes slightly smaller because a larger fraction of the disk matter falls into the black hole, resulting in slight shortening until the freeze-out of the weak interaction is reached. An alternative possibility is that a difference in the initial profile of the disk is reflected. However, the difference in the $Y_{e}$ distribution is quite small, and hence we may conclude that the difference of the spin is not very important for the properties of the viscosity-driven ejecta, as long as we focus on the astrophysically plausible values of $\chi$.

Figure 11(d) compares the mass histogram for models $\mathrm{K} 8$ and $\mathrm{K} 8 \mathrm{n}$ in order to understand the unimportance of the neutrino irradiation for the viscosity-driven ejecta in the black hole-disk system. This figure shows that the two results are quite similar to each other, and hence the neutrino irradiation indeed does not play an important role. This is quite reasonable in our viscous evolution models, because the mass ejection is activated only after the neutrino luminosity drops (i.e., after the weak interaction freezes out), whereas the neutrino irradiation could be 

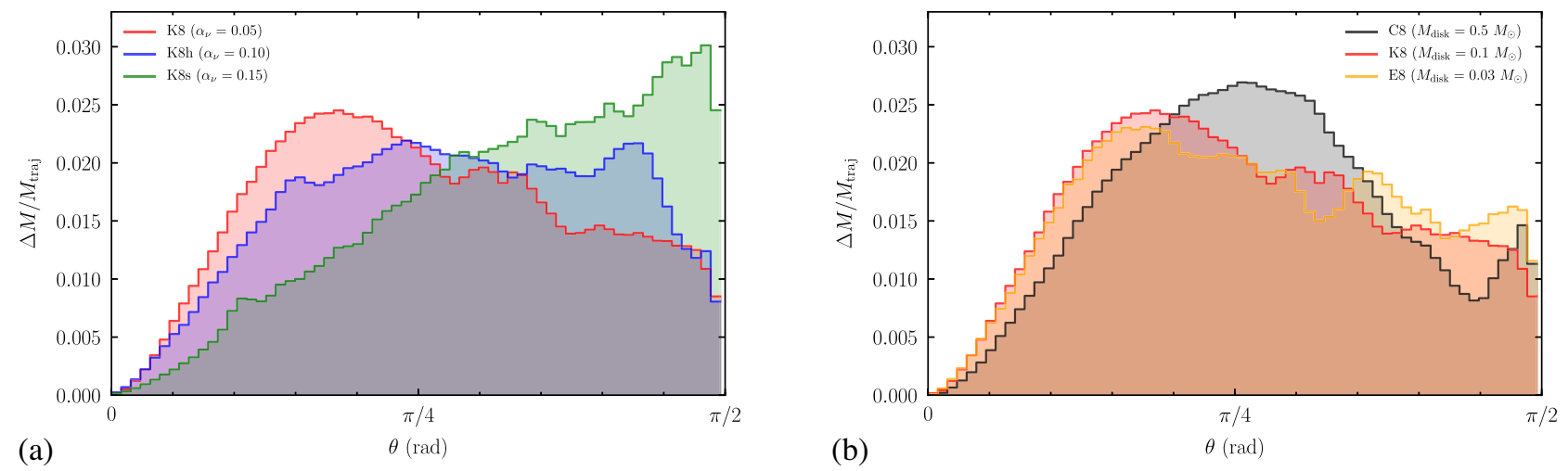

FIG. 12. Mass fraction as a function of the polar direction of the mass ejection (a) for models K8, K8h, and K8s and (b) for models C8, $\mathrm{K} 8$, and E8.

important only for the case that the neutrino luminosity is still high. Thus, the neutrino irradiation would be important only for the case that mass ejection occurs (by a nonviscous effect) in an early stage in which the neutrino luminosity is still high (e.g., Ref. [19]).

Figure 11(e) compares the mass histogram for models $\mathrm{C} 8, \mathrm{~K} 8$, and E8-different initial disk mass models. It is found that for higher disk masses, the lowest end of $Y_{e}$ is larger. As already mentioned in the previous section, for higher disk masses, the time at which the weak interaction freezes out comes later, and the value of $Y_{e}$ for the disk component becomes higher. Associated with this effect, the lowest value of $Y_{e}$ in the ejecta component also increases for larger-disk-mass models. This result suggests that for the case that the remnant disk mass is large-e.g., in the remnant of stellar core collapse to a spinning black hole [48] - the ejecta from the disk may not be very neutron rich and cannot synthesize a substantial fraction of heavy elements like lanthanide (see Sec. III E).

Figure 12 displays the mass fraction as a function of the polar angle of the mass ejection for models $\mathrm{K} 8, \mathrm{~K} 8 \mathrm{~h}$, and K8s [Fig. 12(a)] and for models C8, K8, and E8 [Fig. 12(b)]. This shows that the mass ejection occurs over a wide range of angles except for the direction of the rotation axis, irrespective of the viscous coefficient and disk mass. The absence of mass ejection toward the rotational axis is natural, because the ejecta, which is driven in a region far from the black hole, has a substantial angular momentum. An interesting point is that the primary direction of the mass ejection depends on the magnitude of the viscous coefficient. For $\alpha_{\nu}=0.05$, the mass ejection occurs most strongly toward the direction $\theta \sim \pi / 6$, where $\theta$ denotes the polar angle. However, with the increase of $\alpha_{\nu}$, the primary angle of the mass ejection increases, and for model K8s, the mass ejection occurs primarily toward the direction of the equatorial plane, $\theta \sim \pi / 2$. This indicates that not only the convective activity but also the outward angular momentum transport plays an important role for the mass ejection with larger values of $\alpha_{\nu}$.

\section{E. Nucleosynthesis in the ejecta}

A nucleosynthesis calculation is performed for models $\mathrm{K} 8, \mathrm{~K} 8 \mathrm{~h}, \mathrm{~K} 8 \mathrm{~s}, \mathrm{E} 8$, and C8. For each model, a few tens of thousands of tracer particles are arranged by the method described in Ref. [21], and in each tracer particle, the nucleosynthesis is explored as a post-processing step by using the reaction network code rNET [49]. The reaction network consists of 6300 species from single neutrons and protons up to the isotopes with $Z=110$ (see Ref. [49] for more details). For those relevant to the $r$ process, the rates of both neutron capture (TALYS [50]) and $\beta$ decay (GT2 [51]) are based on the microscopic prediction of nuclear masses, HFB-21 [52]. In this work, we exclude fission process from the network, which is relevant only for $Y_{e}<0.15$. Neutrino-induced reactions are not included, either, which are unimportant, as described in previous sections. Each nucleosynthesis calculation is started when the temperature decreases to $10^{10} \mathrm{~K}$ with the initial compositions of the free neutron and proton being $1-Y_{e}$ and $Y_{e}$, respectively. Note that nuclear statistical equilibrium (NSE) is immediately established at such high temperatures.

Figure 13 displays the mass fraction of each element as a function of the atomic mass number, $A$. In our ejecta data, the peak in $Y_{e}$ comes around $Y_{e} \sim 0.25-0.3$, and the mass fraction with $Y_{e} \lesssim 0.2$ is tiny except for model K8s (see Fig. 11). For such mass distribution with respect to $Y_{e}$, the heavy elements with $A \gtrsim 130$ are not synthesized substantially. By contrast, elements with $A \sim 80-90$ and $A \sim 100-110$ are synthesized significantly through the nucleosynthesis process. The resulting abundance pattern is different from the solar abundance pattern of $r$-process elements [53]. This suggests that low-mass black holedisk systems might be a subdominant site for the $r$-process nucleosynthesis, assuming that the solar abundance pattern is universal [54] in the Universe and that the viscosity-driven mechanism is the main channel of the mass ejection.

For model K8 (as well as model C8), the mass fraction of lanthanide elements is quite small (see the last column of 

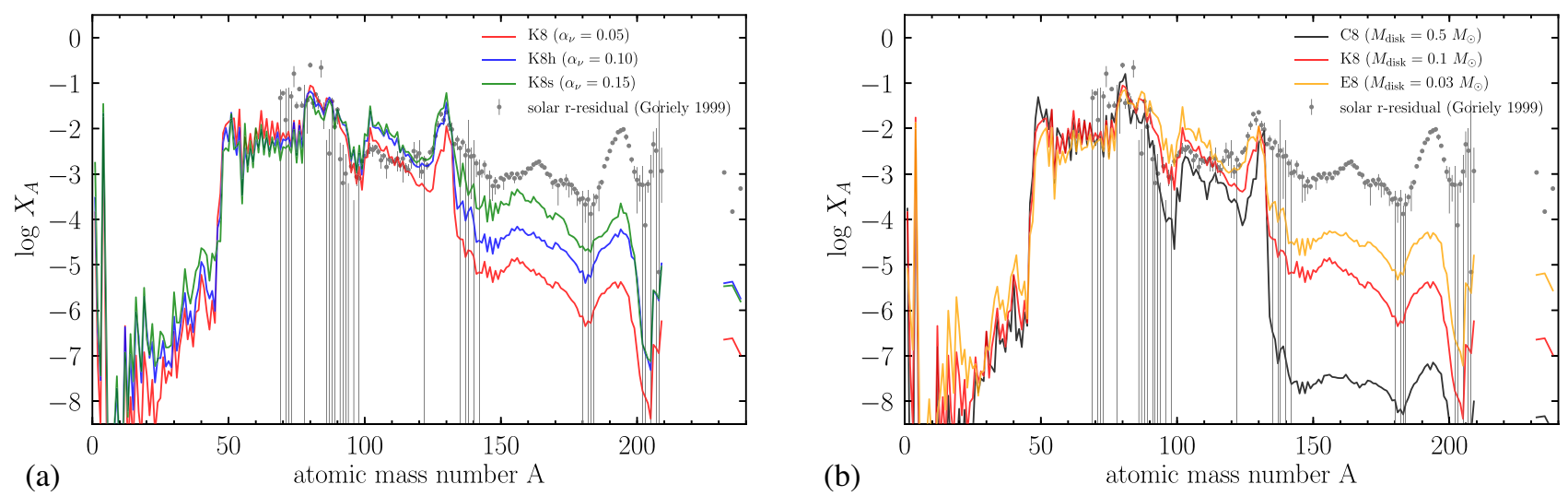

FIG. 13. Patterns of mass fraction obtained in the nucleosynthesis calculation for the ejecta (a) for models K8, K8h, and K8s and (b) for models C8, K8, and E8. The filled circles with error bars denote the $r$-process residual to the Solar System abundance for $A \geq 69$ [53]. The data are shifted to match the calculated mass fraction for model $\mathrm{K} 8$ at $A=83$.

Table II), as expected from the mass histogram shown in Fig. 11. By contrast, for the higher-viscous-coefficient cases (models $\mathrm{K} 8 \mathrm{~h}$ and $\mathrm{K} 8 \mathrm{~s}$ ), a fraction of the lanthanide elements is synthesized, although the amount of the lanthanide and heavier elements is still smaller than that in the solar abundance. The reason for this dependence is that for the higher viscous coefficients, the ejecta contains a fraction of neutron-rich components with $Y_{e} \lesssim 0.2$ due to the earlier mass ejection [see Fig. 11(a)]. Thus, the mass fraction of the lanthanide depends on the magnitude of the viscous coefficient, or in other words, on the onset time of the mass ejection.

For model K8s, the mass fraction of the lanthanide synthesized is about $0.84 \%$, while for models $\mathrm{K} 8$ and $\mathrm{K} 8 \mathrm{~h}$, it is only $0.03 \%$ and $0.16 \%$, respectively. For a small lanthanide fraction, the opacity of the ejecta is not extremely enhanced, whereas for a lanthanide fraction of $\gtrsim 10 \%$, the opacity is $\sim 10^{2}$ times higher than that for lighter elements like iron [55-57]. Our present results suggest that even for the ejecta from a black hole-disk system, the lanthanide fraction could be minor, in contrast to the previous understanding (e.g., Refs. $[10,11,14])$, if the mass ejection sets in for $\gtrsim 0.3 \mathrm{~s}$ after the merger: A kilonova associated with the ejecta from black hole-disk systems may shine in an optical band for the early time after the merger (at $\sim 1 \mathrm{~d}$ ) in contrast to the previous belief [58]. On the other hand, for the high-viscosity model K8s, the mass ejection occurs earlier than for lower-viscosity models so that the mass fraction of the lanthanide can be $\sim 1 \%$. For this case, the enhancement of the opacity would not be negligible [58]. This obviously shows it is very important to quantitatively understand the typical onset time of the mass ejection.

In the astrophysical context, the actual viscous effects should result effectively from turbulence induced by magnetohydrodynamics processes. This implies that for more physical modeling of the mass ejection, we need a magnetohydrodynamics simulation that can accurately capture the nature of the turbulence. For preserving the turbulence in the disk orbiting a stellar-mass black hole in magnetohydrodynamics simulations for seconds, we have to perform a high-resolution nonaxisymmetric simulation. Here, the high resolution implies that both the black-hole spacetime and inner region of the disk are well resolved; in particular, the fastest-growing mode of the MRI has to be resolved, and in addition, the resulting turbulence has to be maintained with a sufficient grid resolution for the entire disk evolution $[26,59,60]$. Although such a simulation is much more expensive than that in the axisymmetric viscous hydrodynamics simulation that we performed in this work, it is not totally impossible in the current computational resources to perform a small number of the simulations (e.g., Refs. [16,18]). We plan to perform a general relativistic neutrino-radiation magnetohydrodynamics simulation to understand the magnitude of the effective viscous coefficient in future work.

Figure 13(b) displays the nucleosynthesis results for models $\mathrm{C} 8, \mathrm{~K} 8$, and $\mathrm{E} 8$. This shows that for more massive disk models, the lanthanide fraction is smaller, as expected from Fig. 11(e). A remarkable point is that for model C8, only a tiny fraction of the heavy elements with $A>132$ like lanthanide are synthesized (see also Table II). This result suggests that if the viscous process is the dominant mechanism of mass ejection, and if the viscous coefficient is not extremely large, the ejecta from the massive disk around black holes may not be the source for the $r$-process nucleosynthesis of the heavy elements like lanthanide and third-peak elements (e.g., gold). Reference [61] has recently illustrated that if the mass ejection sets in within $\sim 100 \mathrm{~ms}$ after the merger, in which the neutrino luminosity is still high, the neutron richness of the ejecta is significantly decreased by the neutrino irradiation. For the massive disks (Ref. [61] considered a disk of mass $\ll 0.1 M_{\odot}$ ), the neutrino luminosity should be higher, as our present work shows, and hence the neutrino irradiation would be even more enhanced. Thus, if the mass ejection 
occurs at a late time (only in the presence of viscous mass ejection), the weak interaction process in the disk enhances the value of $Y_{e}$, and if the mass ejection occurs at an early time (by some powerful process; e.g., by a poloidal magnetic field aligned with the black-hole spin direction), the neutrino irradiation process enhances the value of $Y_{e}$. This suggests that for the massive disk around a black hole to be a site for the nucleosynthesis of the heavy $r$-process elements, a fine-tuning for the timing of the mass ejection would be necessary.

\section{F. Convergence on the grid resolution}

Before closing Sec. III, we comment on the convergence of our numerical results with respect to the grid resolution. As found in Fig. 6(a), the accuracy for following the evolution of rapidly spinning black holes depends strongly on the grid resolution. Due to the truncation error, the black-hole spin spuriously decreases, and the decrease rate can be too high for insufficient grid resolutions to be acceptable. For $\Delta x=0.020 M_{\mathrm{BH}}$ of model K8, the dimensionless spin decreases by $\approx 0.06$ in $1 \mathrm{~s}$. Such a large error is not acceptable. However, the decrease rate is suppressed significantly by improving the grid resolution. For $\Delta x=$ $0.016 M_{\mathrm{BH}}$ and $0.0133 M_{\mathrm{BH}}$, the decrease rates are $\sim 0.02 / \mathrm{s}$ and $0.01 / \mathrm{s}$, respectively, so that the effect of the rapid spin for these simulations can be taken into account for $t \lesssim 2 \mathrm{~s}$ during which the mass ejection is driven.

In Figs. 7 and 10, the time evolution of various quantities is also compared for model $\mathrm{K} 8$ with two different grid resolutions. These figures show that the quantities for the matter outside the black hole and ejecta well achieve convergence with respect to the grid resolution. All these results confirm that our present choice of the grid resolution with $\Delta x=0.016 M_{\mathrm{BH}}$ is acceptable for a reliable numerical simulation.

Figure 11(f) compares the mass histogram as a function of $Y_{e}$ for model $\mathrm{K} 8$ with two different grid resolutions. We find that the two histograms agree broadly with each other, although the mass fraction at each value of $Y_{e}$ does not exactly agree.

\section{SUMMARY}

This paper presents our first numerical results for a viscous neutrino-radiation hydrodynamics simulation of accretion disks surrounding a spinning black hole in full general relativity as models for the evolution of a merger remnant of massive binary neutron stars or low-mass black hole-neutron star binaries. We reconfirm the following results found by previous viscous hydrodynamics studies by other groups [10,14]: About $15 \%-30 \%$ of the disk mass is ejected from the system with the average velocity of $\sim 5 \%-10 \%$ of the speed of light for the plausible profile of the disks as merger remnants. In our simulation, the main driving force of the mass ejection is the viscous heating in the innermost region of the disks and the resulting convection that starts relatively in a late stage of the disk evolution, as well as the viscous angular momentum transport. Our most important conclusion is that for the not extremely high viscous coefficient case, the neutron richness of the ejecta does not become very high, because the weak interaction in the disk enhances the electron fraction during its viscous expansion until the weak interaction freezes out and mass ejection sets in. This results in the suppression of the lanthanide synthesis in the ejecta, and as a result, the opacity of the ejecta may not be very high even for the ejecta from a black hole-disk system [55-57]. A kilonova associated with the ejecta from black hole-disk systems may shine in an optical band for an early stage (e.g., at $\sim 1 \mathrm{~d}$ after the merger) [62].

As we described in Sec. I, in the popular interpretation, we believe that a massive neutron star was temporarily formed after the merger of a binary neutron star in the GW170817 event [3-6]. The reason for this interpretation is that in the presence of the massive neutron star, the system can have a strong and long-lasting neutrino irradiation source, by which the electron fraction of the ejecta can avoid being neutron rich, its opacity can be decreased significantly, and the ejecta can shine for an early stage in the optical band. The last point agrees with the observational results. However, the results of our present simulation suggest that if the mass ejection from the disk surrounding a spinning black hole occurs after a long-term viscous expansion of the disk (for a duration longer than several hundreds of milliseconds) in which the weak interaction plays an important role, the ejecta can be weakly neutron rich and avoid synthesizing a lot of lanthanide elements. For producing such weakly neutronrich ejecta, the mass ejection process must not be efficient in $\lesssim 0.1 \mathrm{~s}$ after the formation of accretion disks.

We also find that the total mass, the average velocity, and the electron fraction of the ejecta depend on the magnitude of the viscous coefficient. For the higher viscous coefficients, the mass ejection sets in earlier, its mass and velocity are larger, and the electron fraction is smaller for the ejecta. By contrast, we find that the quantitative properties of the ejecta depend only weakly on the initial profile of the density, angular velocity, and electron fraction for the disk, as well as the black-hole spin (of astrophysically plausible values).

For high-mass disks, the viscous expansion timescale is increased due to an enhanced dissipation by the neutrino emission (i.e., the timescale until the freeze-out of the weak interaction becomes longer), and hence the electron fraction of the ejecta becomes larger. Thus, for synthesizing heavy elements, lower-mass disks (as well as high viscous coefficients) are favorable. Our result suggests that for the case that the remnant disk mass is large-e.g., in the remnant of stellar core collapse to a spinning black hole [48] — the ejecta from the disk may not be so neutron 
rich that the matter in the ejecta cannot synthesize a substantial fraction of heavy elements like lanthanide. If a mechanism that ejects matter in a short timescale is present, a substantial fraction of neutron-rich matter could be ejected [63]. Magnetohydrodynamics effects may be the mechanism for the efficient mass ejection, but at present, it is not very clear whether this is indeed the case, because the results of the magnetohydrodynamics simulation depend very strongly on the initial condition of the magnetic field strength and profile [18], and long-term magnetohydrodynamics simulations with a variety of the initial magnetic field profiles have not yet been performed while taking into account detailed microphysics (equation of state and neutrino processes).

In the present work, the viscous effect is the driving force for the mass ejection. In reality, the viscous effects should result from turbulence induced by magnetohydrodynamics processes in the astrophysical context. This implies that for more physical modeling of the mass ejection, we obviously need a magnetohydrodynamics simulation. It should be particularly emphasized that the onset time of the mass ejection essentially determines the neutron richness and resulting lanthanide fraction of the ejecta. We find that if the onset time is longer than $\sim 0.3 \mathrm{~s}$, the lanthanide synthesis is significantly suppressed. Magnetohydrodynamics simulations show that early mass ejection is possible if a strong poloidal magnetic field is present at the formation of the disk $[15,16,18]$. However, it is not clear at all whether such a magnetic field favorable for the early mass ejection is present for the remnant disk of a neutron-star merger. A magnetohydrodynamics simulation from the merger stage throughout the postmerger stage is required.

For enhancing and preserving the turbulence in the disk orbiting a stellar-mass black hole in magnetohydrodynamics simulations for seconds, we have to perform a highresolution nonaxisymmetric simulation. Such simulations have not been done yet (but see Refs. [16,18]), since the high-resolution radiation magnetohydrodynamics simulation in general relativity is much more expensive than that in the axisymmetric viscous hydrodynamics simulation that we performed in this work. However, it is not impossible in the current computational resources to perform a small number of the simulations. We plan to perform a general relativistic neutrino-radiation magnetohydrodynamics simulation to examine the difference between the viscous effect and the magnetohydrodynamics effect and to understand the magnitude of the effective viscous coefficient in future work. If we can determine the magnitude of the effective viscous coefficient in the magnetohydrodynamics simulation, the results of the viscous hydrodynamics simulations can be used more robustly for predicting the nucleosynthesis in the ejecta from the black hole-disk system.

\section{ACKNOWLEDGMENTS}

We thank Kyohei Kawaguchi and Masaomi Tanaka for helpful discussions. This work was in part supported by Grant-in-Aid for Scientific Research (Grants No. JP16H02183, No. JP16H06342, No. JP17H01131, No. JP18H01213, No. JP18H04595, No. JP18H05236, No. JP19K14720, No. JP16K17706, No. JP16H06341, No. JP17H06363, and No. JP18K03642) of Japanese MEXT/JSPS. We also thank the participants of a workshop entitled "Nucleosynthesis and electromagnetic counterparts of neutron-star mergers" at Yukawa Institute for Theoretical Physics, Kyoto University (No. YITP-T-18-06) for many useful discussions. Numerical computations were performed on Sakura and Cobra clusters at Max Planck Computing and Data Facility, on Oakforest-PACS at the Information Technology Center of the University of Tokyo, on XC50 at the National Astronomical Observatory of Japan, and on XC40 at the Yukawa Institute for Theoretical Physics.

\section{APPENDIX A: WEAK INTERACTION IN THE DISK}

In this appendix, we describe how the electron fraction in the disk is determined by the weak interaction processes. Here, we consider the electron/positron capture on nucleons and nuclei as the major reaction that determines the electron fraction. We suppose that the neutrino absorption process plays only a minor role for the evolution of black hole-disk systems, in particular for the late stage of the disk evolution, because its luminosity is too low to significantly change the electron fraction in the disk.

The reaction rates of the electron and positron capture on free nucleons and heavy nuclei are written as (see, e.g., Ref. [64])

$$
\begin{aligned}
& \mathcal{R}_{\mathrm{ec}}=X_{\mathrm{p}} \lambda_{\mathrm{ec}}^{\mathrm{f}}+\frac{X_{\mathrm{h}}}{\langle A\rangle_{\mathrm{h}}} \lambda_{\mathrm{ec}}^{\mathrm{h}}, \\
& \mathcal{R}_{\mathrm{pc}}=X_{\mathrm{n}} \lambda_{\mathrm{pc}}^{\mathrm{f}}+\frac{X_{\mathrm{h}}}{\langle A\rangle_{\mathrm{h}}} \lambda_{\mathrm{pc}}^{\mathrm{h}},
\end{aligned}
$$

where $X_{\mathrm{n}}, X_{\mathrm{p}}$, and $X_{\mathrm{h}}$ are the mass fractions of neutrons, protons, and heavy nuclei, $\langle A\rangle_{\mathrm{h}}$ is the average mass number of heavy nuclei, and $\lambda_{\mathrm{ec} / \mathrm{pc}}^{\mathrm{f}}$ is the rate of each reaction, which has the unit of $(\text { time })^{-1}$. Here, the superscripts " $\mathrm{f}$ " and "h" indicate the capture on free nucleons and heavy nuclei, respectively. The reaction rates are written by integration with respect to the energy as [65]

$$
\begin{aligned}
\lambda_{\mathrm{ec} / \mathrm{pc}}^{\mathrm{f} / \mathrm{h}}= & \frac{\ln 2}{\langle f t\rangle^{\mathrm{f} / \mathrm{h}} m_{e}^{5} c^{10}} \int_{0}^{\infty} d \omega \omega^{2}\left(\omega-Q_{\mathrm{ec} / \mathrm{pc}}^{\mathrm{f} / \mathrm{h}}\right)^{2} \\
& \times \sqrt{1-\frac{m_{e}^{2} c^{4}}{\left(\omega-Q_{\mathrm{ec} / \mathrm{pc}}^{\mathrm{f} / \mathrm{h}}\right)^{2}}} F_{e}\left(\omega-Q_{\mathrm{ec} / \mathrm{pc}}^{\mathrm{f} / \mathrm{h}}\right) \\
& \times \Theta\left(\omega-Q_{\mathrm{ec} / \mathrm{pc}}^{\mathrm{f}}-m_{e} c^{2}\right),
\end{aligned}
$$


where $\langle f t\rangle$ is the so-called $f t$-value of these reactions, $F_{e}$ is the distribution function of electrons, which is assumed to be the Fermi-Dirac form, and $Q$ is the $Q$-value of these reactions. For the capture processes by free nucleons, $\langle f t\rangle_{\mathrm{ec} / \mathrm{pc}}^{\mathrm{f}} \approx 1035 \mathrm{~s}, \quad Q_{\mathrm{ec}}^{\mathrm{f}}=\left(m_{\mathrm{p}}-m_{\mathrm{n}}\right) c^{2}$, and $Q_{\mathrm{pc}}^{\mathrm{f}}=\left(m_{\mathrm{n}}-m_{\mathrm{p}}\right) c^{2}$. For the capture processes by heavy nuclei, we follow the approximations in Ref. [64] for $f t$-values and $Q$-values with

$$
\begin{aligned}
& Q_{\mathrm{ec}}^{\mathrm{h}}=\mu_{\mathrm{p}}-\mu_{\mathrm{n}}, \\
& Q_{\mathrm{pc}}^{\mathrm{h}}=\mu_{\mathrm{n}}-\mu_{\mathrm{p}},
\end{aligned}
$$

and

$$
\begin{aligned}
& \log _{10}\left(\langle f t\rangle_{\mathrm{ec} / \mathrm{pc}}^{\mathrm{h}} / \mathrm{s}\right) \\
& = \begin{cases}3.2 & \text { (unblocked and } \left.\mu_{e}<Q_{\mathrm{ec} / \mathrm{pc}}\right) \\
2.6 & \text { (unblocked and } \left.\mu_{e}>Q_{\mathrm{ec} / \mathrm{pc}}\right) \\
2.6+25.9 / T_{9} & \text { (blocked), }\end{cases}
\end{aligned}
$$

where $T_{9}=T / 10^{9} \mathrm{~K} . \mu_{n}$ and $\mu_{p}$ denote the relativistic (including the mass) chemical potential of neutrons and protons, respectively. Here, "blocked" and "unblocked" cases imply that $\langle N\rangle \geq 40$ or $\langle Z\rangle \leq 20$ and that $\langle N\rangle<40$ and $\langle Z\rangle>20$, respectively. $\langle N\rangle$ and $\langle Z\rangle$ denote the average neutron and proton numbers of the heavy nuclei, respectively. Note that we do not consider the updates for the electron/positron capture on heavy nuclei in Ref. [66], which play a minor role in the present case owing to the freeze-out of weak interaction at sufficiently high temperatures (see below).

The reaction rates are functions of $\rho, T$, and $Y_{e}$. We can derive the electron fraction in the equilibrium, $Y_{e, \text { eq }}$, at each density and temperature by equating the electron and positron capture rates as

$$
\begin{aligned}
& \mathcal{R}_{\mathrm{ec}}^{\mathrm{f}}\left(\rho, T, Y_{e, \text { eq }}\right)+\mathcal{R}_{\mathrm{ec}}^{\mathrm{h}}\left(\rho, T, Y_{e, \text { eq }}\right) \\
& =\mathcal{R}_{\mathrm{pc}}^{\mathrm{f}}\left(\rho, T, Y_{e, \text { eq }}\right)+\mathcal{R}_{\mathrm{pc}}^{\mathrm{h}}\left(\rho, T, Y_{e, \text { eq }}\right) .
\end{aligned}
$$

Here we do not consider the blocking by neutrinos, because it is important only for the case that the optical depth to neutrinos is large. Note that the disk material is supposed to be optically thin to neutrinos except for the very early epoch of the disk evolution (i.e., $t \lesssim 0.1 \mathrm{~s}$ ).

Figure 14 plots the contour of $Y_{e, \text { eq }}$ (black curves) together with the distribution of $Y_{e}$ for the disk material (color plots) in the $\rho-T$ plane at selected time slices, $t=0,0.03,0.1$, and $0.3 \mathrm{~s}$ of the simulation for model $\mathrm{K} 8$. Here, we employ the DD2 equation of state to determine the mass fractions, average mass number, and chemical potentials in Eqs. (A1)-(A3) as functions of $\rho, T$, and $Y_{e}$. Due to the viscous heating/angular momentum transport and resulting expansion, the density and temperature in the disk decrease. On the other hand, the specific entropy increases. Thus, the distribution of the disk matter moves basically to the bottom-left region gradually. Figure 14 also displays the weak interaction timescale at each point, $t_{\text {eq }}$, which is defined as the inverse of Eq. (A1) with $Y_{e}=Y_{e, \mathrm{eq}}$.

In an early stage of the disk evolution, the condition of $t>t_{\mathrm{eq}}$ is satisfied for the majority of the disk matter so that the values of $Y_{e}$ approach $Y_{e, \text { eq }}$. For $t \lesssim 0.1 \mathrm{~s}$, a part of the disk matter has high density with $\gtrsim 10^{10} \mathrm{~g} / \mathrm{cm}^{3}$, and hence, until $t=0.1 \mathrm{~s}$, we still have the material with $Y_{e}<0.1$. However, because of the expansion of the disk, the density goes below $\sim 10^{10} \mathrm{~g} / \mathrm{cm}^{3}$, and then the values of $Y_{e}$ have to be larger than $\sim 0.2$ at $t=0.3 \mathrm{~s}$. Also, the increase of the entropy by the viscous heating helps the leptonization of the disk.

It is still possible to eject the low- $Y_{e}$ material from the disk in the presence of the mass ejection mechanism with a short timescale of $\lesssim 0.1 \mathrm{~s}$. As discussed in Sec. III D, one possibility is the Lorentz force by the aligned magnetic field, which is not taken into account in this work. If such a mechanism works for $t \lesssim 0.1 \mathrm{~s}$, a fraction of the low- $Y_{e}$ material may be ejected. However, for the purely viscosity-driven mass ejection with the timescale $\gtrsim 0.3 \mathrm{~s}$, the ejecta cannot have a small value of $Y_{e} \lesssim 0.2$. This agrees totally with the results of our numerical simulations.

The dependence of $t_{\mathrm{eq}}$ on the temperature shows that for $k T \gtrsim 2 \mathrm{MeV}, t_{\text {eq }} \lesssim 0.2 \mathrm{~s}$ and for $k T \gtrsim 1.4 \mathrm{MeV}, t_{\text {eq }} \lesssim 1 \mathrm{~s}$. Thus, when the temperature decreases below $\sim 2 \mathrm{MeV} / k$, the neutrino cooling timescale, which has the same order of magnitude as $t_{\mathrm{eq}}$ in the region where the baryon dominates the internal energy, becomes longer than the viscous timescale, and as a result, the freeze-out of the weak interaction occurs. This effect is also observed in our numerical results. 

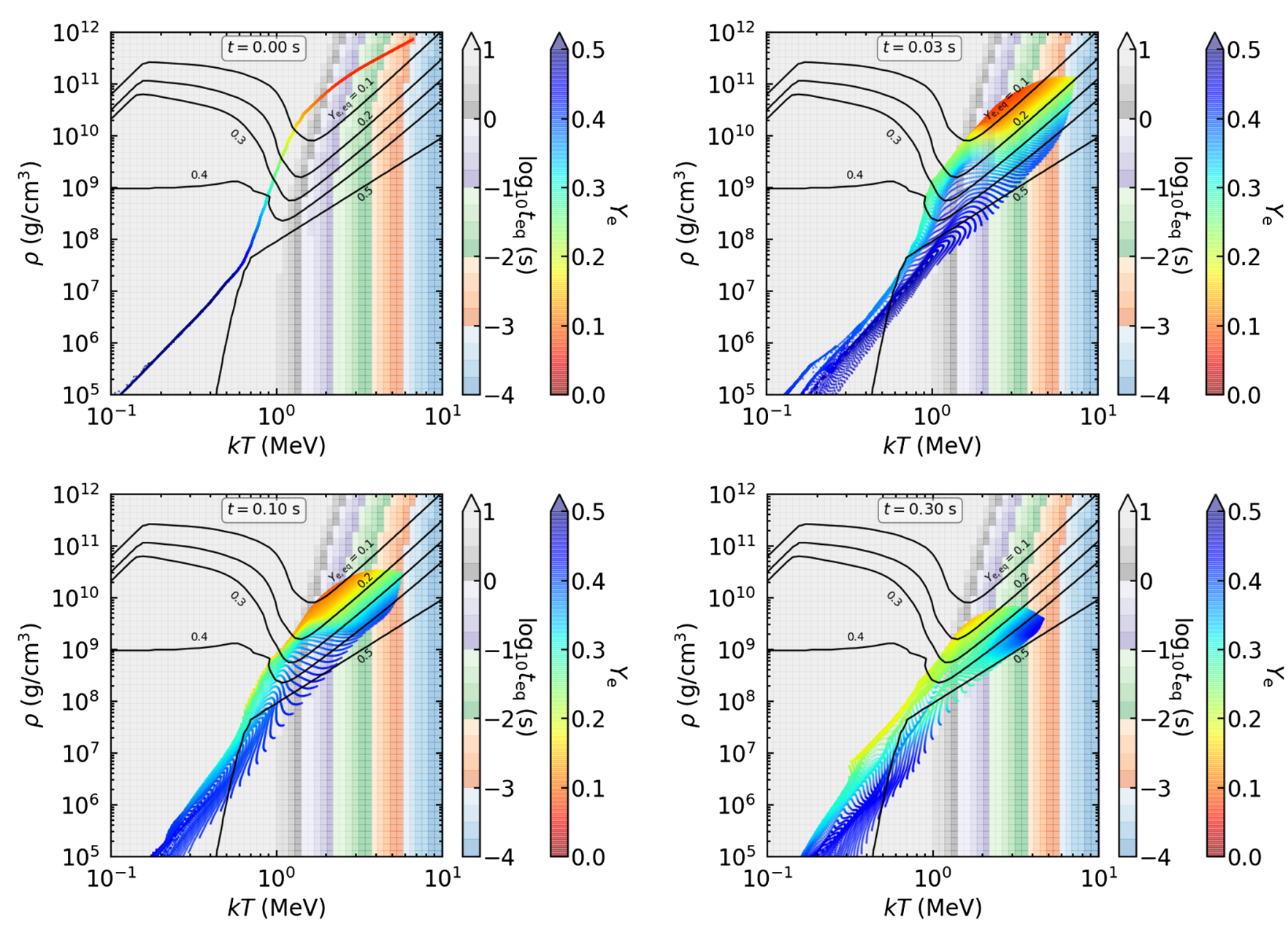

FIG. 14. Distribution of disk matter in the $\rho$ - $T$ plane for model K8 at $t=0$ (upper left), $0.03 \mathrm{~s}$ (upper right), $0.1 \mathrm{~s}$ (lower left), and $0.3 \mathrm{~s}$ (lower right). Each point shows the density and temperature at a certain grid point in the simulation result. $Y_{e}$ at each grid point is displayed by the color. The black curves show the contours of $Y_{e, \text { eq. }}$. Behind the $\rho-T$ distribution of the simulation, we also plot (by shaded color) the weak interaction timescale at each bin, $t_{\text {eq }}$, which is defined as the inverse of Eq. (A1) with $Y_{e}=Y_{e \text { eeq }}$ In the plane, there is a region for which $Y_{e, \text { eq }}$ should be higher than 0.6 [65]. We cannot calculate the electron/positron capture rates in such a region because $Y_{e}>0.6$ is out of range of the tabulated equation of state, and thus the timescale there is defined as the geometric mean of Eqs. (A1) and (A2), with $Y_{e}=0.6$.

\section{APPENDIX B: DEPENDENCE ON THE DISK COMPACTNESS}

To show the dependence of the viscous evolution on the initial compactness of the disk, we here compare the results for models K8 and D8. Figure 15 displays the evolution of the same quantities for the matter located outside the black hole as in Fig. 9, but for models K8 and D8. It is found that the values of $M_{\text {fall }}$ and $M_{\text {eje }}$ for model D8 are larger and smaller, respectively, than those for model K8. For model D8, the ejecta mass is $\approx 15 \%$ of the initial disk mass, which is appreciably smaller than that for models with a less compact disk like $\mathrm{K} 8$ and $\mathrm{Y} 8$, for which the ejecta mass is $\gtrsim 20 \%$ of the initial disk mass (see Table II).
Because a larger fraction of the disk mass falls into the black hole in the early stage of the viscous evolution, the disk mass for model D8 becomes smaller than for model K8 during the long-term viscous evolution. Due to this, the timescale to reach the freeze-out of the weak interaction for model D8 is slightly shorter than for model K8 (see Sec. III C). However, besides this small difference, the evolution processes for the two models are quite similar to each other. The final average value of $Y_{e}$ for model D8 is only slightly smaller than for model K8. As a result, the average value of $Y_{e}$ and the mass histogram for the ejecta are not very different between the two models. The average velocity of the ejecta is also approximately the same for the two models (see Table II). 

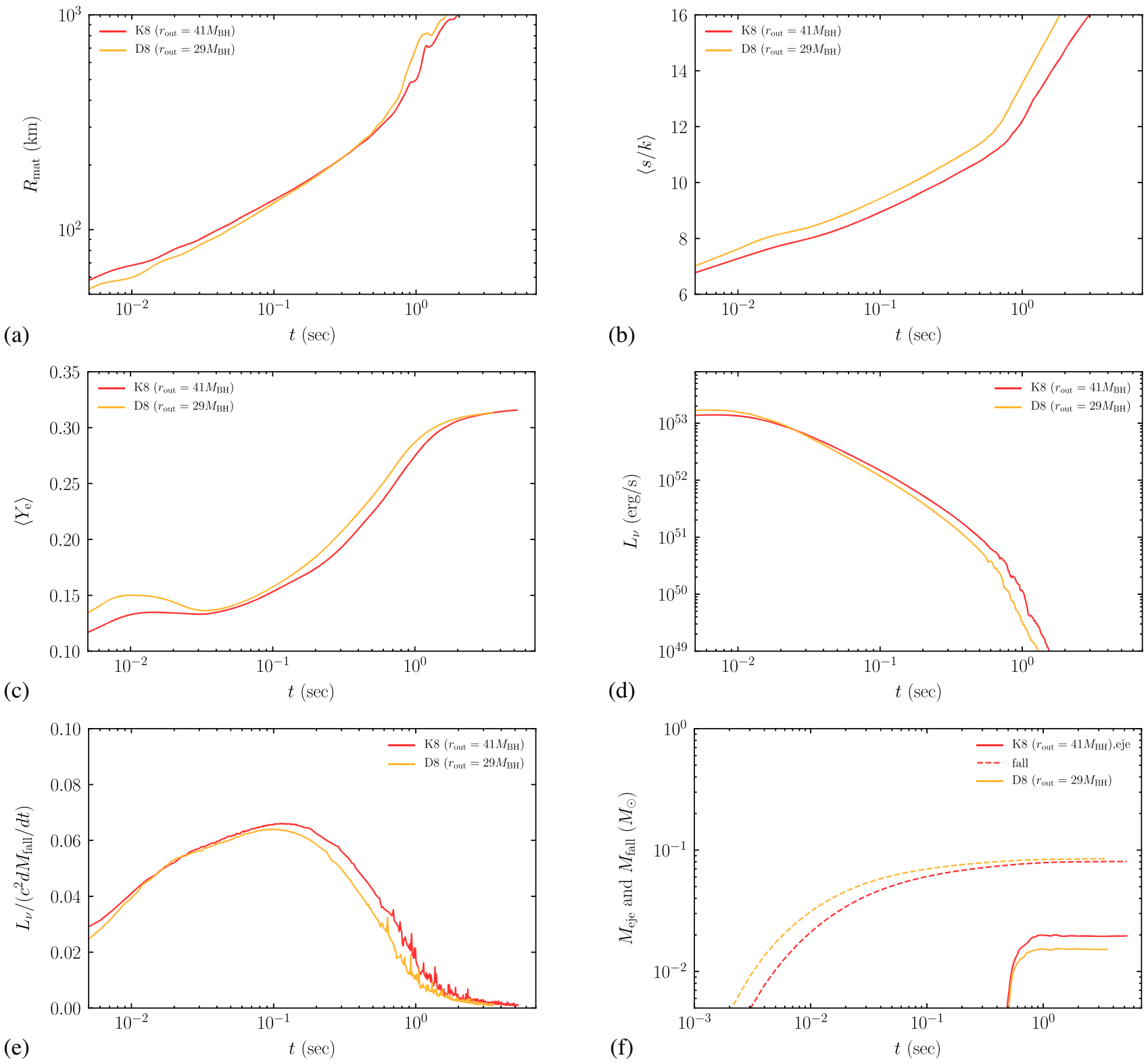

FIG. 15. The same as Fig. 9, but for comparison between models K8 and D8.

[1] B. P. Abbott et al., Phys. Rev. Lett. 119, 116101 (2017).

[2] B. P. Abbott et al., Astrophys. J. 848, L12 (2017).

[3] D. Kasen, B. D. Metzger, J. Barnes, E. Quataert, and E. Ramirez-Ruiz, Nature (London) 551, 80 (2017).

[4] B. Margalit and B. D. Metzger, Astrophys. J. 850, L19 (2017).

[5] M. Shibata, S. Fujibayashi, K. Hotokezaka, K. Kiuchi, K. Kyutoku, Y. Sekiguchi, and M. Tanaka, Phys. Rev. D 96, 123012 (2017).

[6] A. Perego, D. Radice, and S. Bernuzzi, Astrophys. J. 850, L37 (2017).
[7] A Goldstein et al., Astrophys. J. 848, L14 (2017); V. Savchenko et al., Astrophys. J. 848, L15 (2017).

[8] K. P. Mooley, A. T. Deller, O. Gottlieb, E. Nakar, G. Hallinan, S. Bourke, D. A. Frail, A. Horesh, A. Corsi, and K. Hotokezaka, Nature (London) 561, 355 (2018).

[9] B. P. Abbott et al., arXiv:2001.01761.

[10] R. Fernández and B. D. Metzger, Mon. Not. R. Astron. Soc. 435, 502 (2013).

[11] B. D. Metzger and R. Fernández, Mon. Not. R. Astron. Soc. 441, 3444 (2014). 
[12] A. Perego, S. Rosswog, R. M. Cabezon, O. Korobkin, R. Kappeli, A. Arcones, and M. Liebendorfer, Mon. Not. R. Astron. Soc. 443, 3134 (2014).

[13] R. Fernández, D. Kasen, B. D. Metzger, and E. Quataert, Mon. Not. R. Astron. Soc. 446, 750 (2015).

[14] O. Just, A. Bauswein, R. A. Pulpillo, S. Goriely, and H.-T. Janka, Mon. Not. R. Astron. Soc. 448, 541 (2015).

[15] D. M. Siegel and B. D. Metzger, Phys. Rev. Lett. 119, 231102 (2017); Astrophys. J. 858, 52 (2018).

[16] R. Fernández, A. Tchekhovskoy, E. Quataert, F. Foucart, and D. Kasen, Mon. Not. R. Astron. Soc. 482, 3373 (2019).

[17] A. Janiuk, Astrophys. J. 882, 163 (2019).

[18] I. M. Christie, A. Lalakos, A. Tchekhovskoy, R. Fernández, F. Foucart, E. Quataert, and D. Kasen, Mon. Not. R. Astron. Soc. 490, 4811 (2019).

[19] J. M. Miller, B. R. Ryan, J. C. Dolence, A. Burrows, C. J. Fontes, C. L. Fryer, O. Korobkin, J. Lippuner, M. R. Mumpower, and R. T. Wollaeger, Phys. Rev. D 100, 023008 (2019).

[20] S. Fujibayashi, K. Kiuchi, N. Nishimura, Y. Sekiguchi, and M. Shibata, Astrophys. J. 860, 64 (2018).

[21] S. Fujibayashi, S. Wanajo, K. Kiuchi, K. Kyutoku, Y. Sekiguchi, and M. Shibata (to be published).

[22] M. Shibata, Numerical Relativity (World Scientific, Singapore, 2016).

[23] M. Shibata and T. Nakamura, Phys. Rev. D 52, 5428 (1995); T. W. Baumgarte and S. L. Shapiro, Phys. Rev. D 59, 024007 (1998).

[24] M. Campanelli, C. O. Lousto, P. Marronetti, and Y. Zlochower, Phys. Rev. Lett. 96, 111101 (2006); J. G. Baker, J. Centrella, D.-I. Choi, M. Koppitz, and J. van Meter, Phys. Rev. Lett. 96, 111102 (2006).

[25] D. Hilditch, S. Bernuzzi, M. Thierfelder, Z. Cao, W. Tichy, and B. Brügmann, Phys. Rev. D 88, 084057 (2013).

[26] S. A. Balbus and J. F. Hawley, Rev. Mod. Phys. 70, 1 (1998).

[27] M. Alcubierre, B. Brügmann, D. Holz, R. Takahashi, S. Brandt, E. Seidel, and J. Thornburg, Int. J. Mod. Phys. D 10, 273 (2001).

[28] M. Shibata, Prog. Theor. Phys. 104, 325 (2000); Phys. Rev. D 67, 024033 (2003); M. Shibata and Y. Sekiguchi, Prog. Theor. Phys. 127, 535 (2012).

[29] M. Shibata, K. Kiuchi, and Y. Sekiguchi, Phys. Rev. D 95, 083005 (2017).

[30] M. Shibata, Phys. Rev. D 76, 064035 (2007).

[31] J. Karkowski, W. Kulczycki, P. Mach, E. Malec, A. Odrzywolek, and M. Pirog, Phys. Rev. D 97, 104017 (2018).

[32] S. Banik, M. Hempel, and D. Bandyophadyay, Astrophys. J. Suppl. Ser. 214, 22 (2014).

[33] F. X. Timmes and F. D. Swesty, Astrophys. J. Suppl. Ser. 126, 501 (2000).

[34] Y. Sekiguchi, K. Kiuchi, K. Kyutoku, and M. Shibata, Phys. Rev. D 91, 064059 (2015); 93, 124046 (2016).

[35] F. Foucart, R. Haas, M. D. Duez, E. O’Connor, C. D. Ott, L. Roberts, L. E. Kidder, J. Lippuner, H. P. Pfeiffer, and M. A. Scheel, Phys. Rev. D 93, 044019 (2016).

[36] D. Radice, F. Galeazzi, J. Lippuner, L. F. Roberts, C. D. Ott, and L. Rezzolla, Mon. Not. R. Soc. Astron. 460, 3255 (2016).
[37] L. Bovard, D. Martin, F. Guercilena, A. Arcones, and L. Rezzolla, and O. Korobkin, Phys. Rev. D 96, 124005 (2017).

[38] F. Foucart, E. O'Connor, L. Roberts, M. D. Duez, R. Haas, L. E. Kidder, C. D. Ott, H. P. Pfeiffer, M. A. Scheel, and B. Szilagyi, Phys. Rev. D 91, 124021 (2015); F. Foucart, D. Desai, W. Brege, M. D. Duez, D. Kasen, D. A. Hemberger, L. E. Kidder, H. P. Pfeiffer, and M. A. Scheel, Classical Quantum Gravity 34, 044002 (2017).

[39] K. Kyutoku, K. Kiuchi, Y. Sekiguchi, M. Shibata, and K. Taniguchi, Phys. Rev. D 97, 023009 (2018).

[40] J. F. Hawley, Astrophys. J. 381, 496 (1991).

[41] O. Korobkin, E. B. Abdikamalov, E. Schnetter, N. Stergioulas, and B. Zink, Phys. Rev. D 83, 043007 (2011).

[42] K. Kiuchi, M. Shibata, P. J. Montero, and J. A. Font, Phys. Rev. Lett. 106, 251102 (2011).

[43] N. I. Shakura and R. A. Sunyaev, Astron. Astrophys. 24, 337 (1973).

[44] K. Kyutoku, M. Shibata, and K. Taniguchi, Phys. Rev. D 82, 044049 (2010).

[45] J.-L. Tassoul, Theory of Rotating Stars (Princeton University Press, Princeton, NJ, 1978).

[46] J. M. Lattimer and T. J. Mazurek, Astrophys. J. 246, 955 (1981).

[47] W. H. Lee, E. Ramirez-Ruiz, and D. Page, Astrophys. J. 632, 421 (2005).

[48] S. E. Woosley, Astrophys. J. 405, 273 (1993).

[49] S. Wanajo, B. Müller, H.-Th. Janka, and A. Heger, Astrophys. J. 852, 40 (2018).

[50] S. Goriely, S. Hilaire, and A. J. Koning, Astron. Astrophys. 487, 767 (2008).

[51] T. Tachibana, M. Yamada, and Y. Yoshida, Prog. Theor. Phys. 84, 641 (1990).

[52] S. Goriely, N. Chamel, and J. M. Pearson, Phys. Rev. C 82, 035804 (2010).

[53] S. Goriely, Astron. Astrophys. 342, 881 (1999).

[54] J. J. Cowan, C. Sneden, J. E. Lawler, A. Aprahamian, M. Wiescher, K. Langanke, G. Martínez-Pinedo, and F.-K. Thielemann, arXiv:1901.01410.

[55] D. Kasen, N. R. Badnell, and J. Barnes, Astrophys. J. 774, 25 (2013); J. Barnes and D. Kasen, Astrophys. J. 775, 18 (2013).

[56] M. Tanaka and K. Hotokezaka, Astrophys. J. 775, 113 (2013).

[57] M. Tanaka et al., Astrophys. J. 852, 109 (2018).

[58] D. Kasen, R. Fernández, and B. Metzger, Mon. Not. R. Astron. Soc. 450, 1777 (2015).

[59] J. F. Hawley, X. Guan, and J. H. Krolik, Astrophys. J. 738, 84 (2011).

[60] K. Kiuchi, K. Kyutoku, Y. Sekiguchi, and M. Shibata, Phys. Rev. D 97, 124039 (2018).

[61] J. M. Miller et al., arXiv:1912.03378.

[62] K. Kyutoku, S. Fujibayashi, K. Hayashi, K. Kawaguchi, K. Kiuchi, M. Shibata, and M. Tanaka, Astrophys. J. (to be published).

[63] D. M. Siegel, J. Barnes, and B. D. Metzger, Nature (London) 569, 241 (2019).

[64] G. M. Fuller, W. A. Fowler, and M. J. Newman, Astrophys. J. 293, 1 (1985).

[65] A. M. Beloborodov, Astrophys. J. 588, 931 (2003).

[66] K. Langanke and G. Martínez-Pinedo, Nucl. Phys. A673, 481 (2000). 